\title{
Pseudogroupes de Lie de type fini et méthode du repère mobile $\left(^{*}\right)$.
}

\author{
YVON BOSSARD (Rennes)
}

Summary. - We describe precisely the method of moving frames of E. Cartan when, instead of a Lie group, it is a Lie pseudogroup which acts on a manifold. The differentiable groupoïds constitute the natural framework of the theory. The study of the intransitive actions of groups gives the motivations.

\section{Introduction.}

Lorsqu'un groupe de Lie $G$ opère différentiablement sur une variété $V$, il transforme un germe de sous-variété de $V$ en un germe de sous-variété. C'est la caractérisation des orbites pour l'action ainsi définie de $G$ dans l'ensemble des germes de sous-variétés de $V$ qui constitue, on le sait, l'objet de la géométrie différentielle des sous-variétés de $V$ (pour l'action donnée de $G$ sur $V$ ).

Soit $C P^{r}$ l'espace des éléments de contact de dimension $p$ et d'ordre $r$ de la variété $V$ (ef. $I, B, \S 1$ ). L'action de $G$ sur $V$ définit canoniquement une action de $G$ sur $C P^{r}$, en général non transitive. On a donc pour tout $r$ une décomposition de $C P^{r}$ en orbites et l'on sait que la connaissance des fonctions différentiables de $C P^{r}$ dans $R$, constantes sur chacune des orbites, c'est-à-dire des invariants différentiels d'ordre $r$ pour l'action de $G$ sur $V$ permet, dans les cas réguliers, cette caractérisation.

Il est done important d'indiquer un procédé permettant de déterminer explicitement ces invariants différentiels. C'est ici qu'intervient la méthode du repère mobile de E. CARTAX ([2], [5]) qui permet, dans les cas réguliers, de ramener la recherche des invariants différentiels de $C P^{r}$ à la recherche des solutions de systèmes différentiels entièrement explicités.

L'orsqu'au lieu d'un groupe de Lie $G$, e'est un pseudo-groupe de Lie $\Gamma$ qui opère sur $V$, l'action de $T$ sur $V$ se prolonge encore en une action de $\Gamma$ dans l'ensemble des germes de sous-variétés de $V$. Il est possible, lorsque $\Gamma$ est un pseudo-groupe de Lie de type fini d'adapter la méthode du repère mobile à l'étude des orbites définies par $\Gamma$ dans l'ensemble des germes de sous-variétés. C'est en même temps qu'une description précise de la méthode du repère mobile, ce que l'on se propose de montrer dans le présent article.

On s'est systématiquement placé dans le cadre des groupoïdes différentiables, le seul qui nous semble convenir pour une telle étude. Un paragraphe 0 rassemble les résultats relatifs aux groupoïdes, utilisés dans la suite. On démontre au paragraphe $I$

(*) Entrata in Redazione il 12 luglio 1973. 
les théorèmes généraux de la géométrie différentielle des sous-variétés d'une variété $\Gamma$-structurée. La méthode du repère mobile est décrite au paragraphe II.

Par la complexité même des structures de prolongement qui interviennent, la description de la méthode du repère mobile n'est pas immédiate. Son utilité cependant, ne serait-ce que par les beaux exemples traités par E. CARTAN, n'est plus à démontrer.

Mademoiselle P. LIBERMANn m'a beaucoup aidé dans la préparation de ce travail. Je tiens à lui exprimer ici ma reconnaissance.

\section{0. - Groupoïdes differentiables.}

\section{A) Groupoïdes différentiables.}

Définition 1. - Une structure de groupoïde est définie sur un ensemble $\Phi$ par les données suivantes:

1) Un ensemble $B \subset \Phi$, appelé base ou ensemble des unités de $\Phi$.

2) Deux applications $\alpha$ et $\beta$ de $\Phi$ sur $B$ induisant sur $B$ l'application identique, $\alpha$ est l'application source et $\beta$ l'application but.

3) Une application $\mu$, dans $\Phi$, du produit fibré

$$
\Phi_{\substack{\not z \alpha \\ \beta=\alpha}} \Phi=\{(u, v) \in \Phi \times \Phi / \beta(u)=\alpha(v)\}
$$

L'application $\mu$, qui est une loi de composition interne non partout définie, doit (avec la convention $\mu(u, v)=v u$ ) vérifier les conditions suivantes:

a) $\alpha(v u)=\alpha(u) ; \quad \beta(v u)=\beta(v)$;

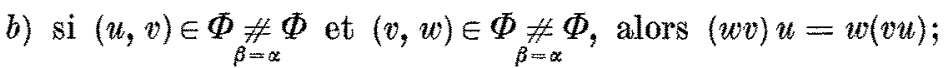

c) $u \alpha(u)=\beta(u) u=u$.

4) Une application $v$ de $\Phi$ dans $\Phi$ vérifiant:

a) $\alpha \circ \nu=\beta ; \quad \beta \circ \nu=\alpha ;$

b) $v(u) u=\alpha(u) ; \quad u v(u)=\beta(u)$.

On pose parfois $v(u)=u^{-1}$.

Un groupoïde est un ensemble $\Phi$ muni d'une structure de groupoïde. 
DÉfINITIon 2. - On dit qu'un groupoïde $\Phi$ est différentiable si:

1) $\Phi$ est une variété différentiable;

2) $B$ est une sous-variété (plongée) de $\Phi$;

3) $\alpha$ est une submersion (et done $\underset{\beta=\alpha}{\not} \Phi$ est une sous-variété de $\Phi \times \Phi$ );

4) $\mu$ et $\nu$ sont différentiables.

En particulier, si $\Phi$ est différentiable, $v$ est un difféomorphisme et $\alpha$ et $\beta$ sont des surmersions (i.e. submersions surjectives).

ExEMrPLE 3. - Soit $G$ un groupe de Lie opérant différentiablement sur une variété $\nabla$ On définit sur $V \times G$ une structure de groupoïde différentiable en posant:

$$
\begin{aligned}
& B=V \times\{\varepsilon\} \quad(\varepsilon=\text { élément neutre de } G) \\
& \alpha(x, g)=(x, \varepsilon) ; \quad \beta(x, g)=(g x, \varepsilon) \\
& \mu[(x, g),(g x, h)]=(x, h g) \\
& v(x, g)=\left(g x, g^{-1}\right)
\end{aligned}
$$

Pour d'autres exemples, voir [8].

On définit aisément la notion de sous-groupoïde $\Phi$ d'un groupoïde $\Psi$ (on impose à l'ensemble des unités de $\Psi$ d'être le même que celui de $\Phi)$. Lorsque $\Phi$ est un groupoïde différentiable, deux cas peuvent se présenter (comme pour les groupes de Lie): le sous-groupoïde $\Psi$ est soit immergé, soit plongé dans $\Phi$. Seul le second cas interviendra dans la suite, done:

DÉFINITION 4. - On dit qu'un sous-groupoïde $\Psi$ d'un groupoïde différentiable $\Phi$ est un sous-groupoïde différentiable, si:

1) $\Psi$ est une sous-variété (plongée) de $\Phi$;

2) $B$ est une sous-variété de $\Psi$;

3) $\alpha$ est une submersion de $\Psi$ sur $B$.

\section{B) Action d'un groupoïde différentiable sur une variété.}

Dans ce paragraphe et dans le suivant, les groupoïdes sont supposés différentiables.

Soient $E$ une variété et $p$ une application différentiable surjective de $E$ sur la variété $B$ des unités du groupoïde $\Phi$. La projection $\alpha: \Phi \rightarrow B$ étant une submersion, le produit fibré

$$
\Phi \underset{\alpha=p}{\not=D}=\{(u, x) \in \Phi \times E / \alpha(u)=p(x)\}
$$

est une sous-variété de $\Phi \times E$. 
Définition 5. - Une action différentiable de $\Phi$ sur $E$ compatible avee $p$ est une application différentiable $\delta$ de $\Phi_{\substack{\not=p \\ \alpha=\emptyset}}^{\not E}$ dans $E$ vérifiant (avec la convention $\delta(u, x)=$ $=u x)$ les conditions suivantes:

1) Si $e \in B$ et $p(x)=e$, on a $e x=x$.

2) Si $(u, v) \in \Phi_{\beta=\alpha}^{\#} \Phi$ et si $(u, x) \in \Phi_{\alpha=p}^{\#} E$, alors :

$$
(v, u x) \in \Phi_{\substack{\not=p \\ \alpha=0}} \quad \text { et } \quad v(u, x)=(v u) x
$$

Définimion 6. - Soient $E$ et $E^{\prime}$ deux variétés et $I$ une submersion de $E$ sur $E^{\prime}$. On dit que des actions $\delta$ et $\delta^{\prime}$ du groupoïde $\Phi$ sur $E$ et $E^{\prime}$ respectivement, sont compatibles avec $\Pi$ si, pour tout $\left(u, x^{\prime}\right)$ appartenant à $\Phi_{\substack{\not \neq \\ z=p^{\prime}}}^{\not E^{\prime}}$, on a:

$$
\Pi \circ \delta^{\prime}\left(u, x^{\prime}\right)=\delta\left(u, \Pi x^{\prime}\right) \text {. }
$$

Définition 7: Etant donné $x \in E$ :

l'orbite de $x$ est le sous-ensemble $O_{x}=\delta\left[x^{-1}(p(x)) \times\{x\}\right]$ des éléments de $E$ de la forme $u x$, avec $u \in \Phi$;

le groupe d'isotropie $\Phi_{x}$ de $x$ est l'ensemble des $u \in \Phi$ tels que $u x=x$.

On note $R_{\Phi}$ la relation d'équivalence: « $x$ et $y$ ont même orbite ». Une partie $F \subset E$ est dite saturée si elle est saturée pour $R_{\Phi}$, e'est-à-dire si c'est une réunion d'orbites.

DÉfInition 8. - Soit $U$ un ouvert saturé de $E$. On dit qu'une submersion $f$ de source $U$ est une $\Phi$-submersion si, pour tout $x \in U$,

$$
O_{x}=\{y \in U / f(y)=f(x)\},
$$

e'est-à-dire si les images réciproques des points du but sont des orbites.

Proposition 9. - Pour un ouvert saturé $U$ de $E$, les deux conditions suivantes sont équivalentes:

1) 11 existe une $\Phi$-submersion de source $U$.

2) La relation $R_{\Phi}$ est régulière dans $U$ (i.e $U / R_{\Phi}$ est une variété et $U \rightarrow U / R_{\Phi}$ est une submersion).

Preuve. - Immédiate à partir des définitions. Remarquer que si $f: U \rightarrow N$ est une $\Phi$-submersion, le but de $f$ est difféomorphe à l'espace des orbites de $\Phi$ dans $U$.

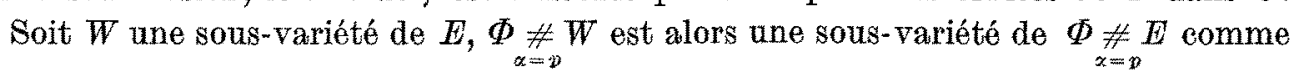
image réciproque de $W$ par la submersion de $\underset{\substack{\not=p \\ \alpha=p}}{\not}$ sur $E$ définie par $(u, x) \rightarrow x$. 
DÉfinition 10. - On dit qu'un ouvert saturé $U$ de $E$ est décomposable s'il existe

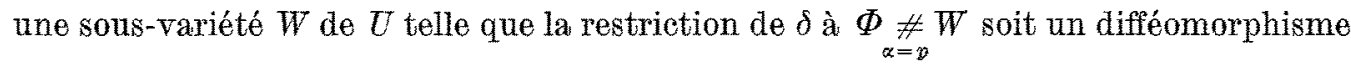

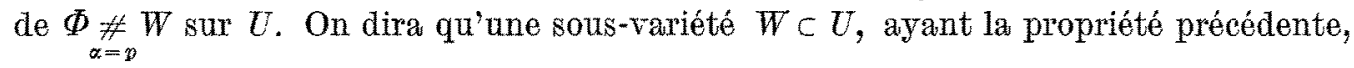
est adaptée à $U$.

Soit $U$ un ouvert décomposable et soit $W$ une sous-variété adaptée à $U$. En composant $\delta^{-1}: U \rightarrow \underset{\alpha=y}{\not \#} W$ avec l'injection canonique de $\Phi_{\alpha=y}^{\# W}$ dans $\Phi \times W$, on obtient une application $\left(\Pi_{1}, \Pi_{2}\right)$ de $U$ dans $\Phi \times W$ qu'on appellera la décomposition de $U$ associée à $W$.

Définition 11. - On dit que $x \in E$ est régulier s'il existe un ouvert décomposable contenant $x$.

Exemple 12. - Considérons l'action, sur $\boldsymbol{R}^{2}$, du groupe des rotations $O^{+}\left(\boldsymbol{R}^{2}\right)$ d'où (cf. exemple 3) une action sur $\boldsymbol{R}^{2}$ du groupoïde $\boldsymbol{R}^{2} \times O^{+}\left(\boldsymbol{R}^{2}\right)$. Pour cette action, l'ouvert $\boldsymbol{R}^{2}-\{0\}$ est décomposable, car il suffit de prendre pour variété $W$ une demidroite ouverte issue de 0 (0 exchus). Tout point de $\boldsymbol{R}^{2}-\{0\}$ est done régulier. L'origine $O$ par contre n'est pas un point régulier, car le groupe d'isotropie en ce point est $O^{+}\left(\boldsymbol{R}^{2}\right)$.

Les actions de proupoïdes que l'on étudie dans la suite, sont obtenues par prolongement de l'action sur une variété d'un groupe ou d'un pseudogroupe de Lie. La condition supplémentaire suivante est alors vérifiée:

Condition A. - Il existe, pour $u \in \Phi$, une section différentiable $\sigma$ de $\alpha$, définie dans un voisinage $U$ de $\alpha(u)$, telle que:

1) $\sigma(\alpha(u))=u$;

2) l'application $s: v \rightarrow \sigma(\beta(v)) v$. définie dans $\beta^{-1}(U) \subset \Phi$ est un difféomorphisme;

3) l'application $\lambda: x \rightarrow \sigma(p(x)) x$ définie dans $p^{-1}(U)$ est un difféomorphisme ${ }^{*}$

Une telle section $\sigma$ sera dite primitive. L'application $\lambda$ (resp. s) est le difféomorphisme de $E$ (resp. $\Phi$ ) associé à $\sigma$.

On suppose désormais que les groupoïdes vérifient la condition $A$.

ExeMPLes 13. - Reprenons l'exemple $3 ; \Phi=V \times G$ opère différentiablement sur $V$ par: $\delta((x, g), x)=g x$.

Si $u=(x, g) \in \Phi$, l'application $\sigma:(y, \varepsilon) \rightarrow(y, g)$ est une section primitive.

Si $T$ est un pseudogroupe de difféomorphismes de $V$, et si $\Phi$ est le groupoïde d'ordre $k$ associé à $\Gamma$ (cf. I), les applieations: $x \rightarrow j_{x}^{k} \varphi[\varphi \in \Gamma)$ sont des sections primitives pour les actions naturelles de $\Phi$ définies au paragraphe I.

L'intérêt des sections primitives provient de ce qu'elles permettent de construire, pour tout $(u, x) \Phi_{\alpha=D}^{\not Z} \boldsymbol{E}$, un difféomorphisme local $\chi$ de $\Phi_{\alpha=y}^{\not \#} \boldsymbol{E}$ défini au voisinage de $(p(x), x)$, transformant $(p(x), x)$ en $(u, x)$ et compatible avec l'action de $\Phi$ sur $E$, Plus précisément: 
Proposition 14. - Soit $(u, x) \in \Phi_{\alpha=p}^{\# E}$ et soit $\sigma$ une section primitive de $\Phi$ telle que $\sigma(\alpha(u))=u$. L'application $\chi$ définie au voisinage de $(p(x), x)$ dans $\underset{\substack{\alpha=x \\ \alpha=p}}{\not E}$ par:

$$
\chi(v, y)=(\sigma(p(v y)) v, y)=(\sigma(\beta(v)) v, y),
$$

est un difféomorphisme à valeurs dans un voisinage de $(u, x)$ tel que:

1) $\chi(p(x), x)=(u, x)$

2) $\delta \circ \chi=\lambda \circ \delta$ ( $\lambda$ difféomorphisme de $E$ associé à $\sigma)$.

Prevve. - La vérification des conditions 1) et 2) est immédiate à partir des définitions. De plus, si $s$ est le difféomorphisme local de $\Phi$ associé à $g$, on $a: \chi^{-1}(w, y)=$ $=\left(s^{-1}(w), y\right)$ et done $\chi$ est un difféomorphisme.

DÉFInITION 15. - On dit qu'une sous-variété $W$ de $E$ est transverse aux orbites de $\Phi$ dans $E$, si pour tout $x \in W$, on a:

$$
T_{x} E=T_{x} W+T_{x} O_{x}
$$

où $O_{x}$ est l'orbite de $x$ dans $E$.

THÉoRÈme 16. - Si $W \subset E$ est une sous-variété transverse aux orbites de $\Phi$ dans $E$,

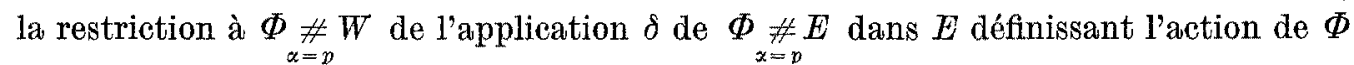
sur $E$ est une submersion.

Preuve. - Soit $\delta_{1}$ la restriction de $\delta$ à $\Phi_{\alpha=p}^{\not \#} W$.

1) $\delta_{1}$ est une submersion en $(p(x), x)$.

En effet, l'espace tangent $T_{x} O_{x}$ appartient évidemment à l'image de $T_{p(\langle x, x)} \delta_{1}$. Il en est de même de $T_{x} W$, car la restriction à $W$ de la section canonique $y \rightarrow$ $\rightarrow(p(y), y)$ de $E$ dans $\underset{\alpha=p}{\not \# E}$ est une section de $\delta_{1}$ transformant $x$ en $(p(x), x)$.

2) $\delta_{1}$ est une submersion en $(u, x)$.

Soit en effet $(u, x) \in \Phi_{\alpha=\nu}^{\not} W$, soit $\sigma$ une section primitive de $\Phi$ tells que $\sigma(\alpha(u))=u$ et soit $\chi$ le difféomorphisme local de $\Phi \underset{\alpha=y}{\#}$ associé à $\sigma$ par la proposition précédente.

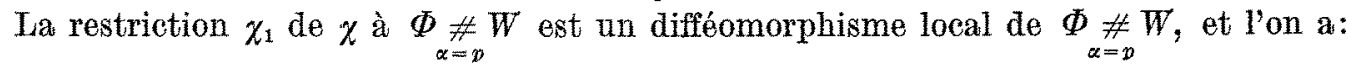
$\delta_{1} \circ \chi_{1}=\lambda_{\circ} \delta_{1}$, on a donc:

$$
\operatorname{Im}\left(T_{(u, x)} \delta_{1}\right)=\operatorname{Im}\left(T_{(p(x), x)}\left(\delta_{1} \circ \chi_{1}\right)=\operatorname{Im} T_{(p(x), x)}\left(\lambda_{0} \circ \delta_{1}\right)=T_{u x} E .\right.
$$

Corollame 17. - Soient $f: U \rightarrow V$ une $\Phi$-submersion, $W$ l'image d'un ouvert de $V$ par une section différentiable de $f$ et $\tilde{U}$ l'ouvert saturé de $W$. Pour que $\tilde{U}$ soit décomposable, il faut et il suffit que les groupes d'isotropie de $\Phi$ dans $\tilde{U}$ se réduisent à l'identité. 
En effet, $W$ est alors transverse aux orbites, la restriction $\delta_{1}$ de $\delta$ à $\underset{\alpha=y}{\# W}$ est done une submersion, et pour que $\delta_{1}$ soit bijective, il faut et il suffit, précisément, que dans $\tilde{U}$, les groupes d'isotropie soient tous l'identité.

Corollatre 18. - Un point $x \in E$ est régulier, si et seulement si il existe un voisinage ouvert $U$ de $x$ tel que

1) $U$ est la souree d'une $\Phi$-submersion;

2) Dans $U$, les groupes d'isotropie de $\Phi$ se réduisent àl'identité.

En effet, le corollaire précédent montre qu'il existe, dans $U$, un voisinage ouvert de $x$ qui est décomposable.

Corollaire 19. - Soient $E$ et $E^{\prime}$ deux variétés, $\Pi$ une submersion de $E^{\prime}$ sur $E$, $\delta$ et $\delta^{\prime}$ des actions de $\Phi$ sur $E$ et $E^{\prime}$ compatibles avec $\Pi$. Pour tout ouvert décomposable $U$ de $E$, l'ouvert $U^{\prime}=\Pi^{-1}(U)$ est décomposable dans $E^{\prime}$.

Soit, en effet, $W$ une sous-variété adaptée à $U ; W$ est transverse aux orbites de $\Phi$ dans $E$. Soit $W^{\prime}=\Pi^{-1}(W)$; la compatibilité de $\delta$ et $\delta^{\prime}$ avec $\Pi$ implique alors successivement que:

1) $U^{\prime}$ est le saturé de $W^{\prime}$ dans $E^{\prime}$;

2) La restriction de $\delta^{\prime}$ à $\Phi_{\alpha=p^{\prime}}^{\not \# y^{\prime}} W^{\prime}$ est bijective (car le groupe d'isotropie de $x^{\prime} \in U^{\prime}$ est contenu dans celui de $P\left(x^{\prime}\right) \in U$, qui est l'identité)

3) $W^{\prime}$ est transverse aux orbites de $\Phi$ dans $E^{\prime}$ car, $I I$ étant une submersion, pour $x^{\prime} \in w^{\prime}, T_{x}, E^{\prime}$ est la somme de $T_{x}, W^{\prime}$ et d'un sous-espace dont la projection par $T_{x}, \Pi$ est $T_{x} O_{x}$ (avec $\left.x=p\left(x^{\prime}\right)\right)$ or, la restriction de $\Pi$ à l'orbite $O_{x}$, est un difféomorphisme de $O_{w^{\prime}}$ sur $O_{x}$
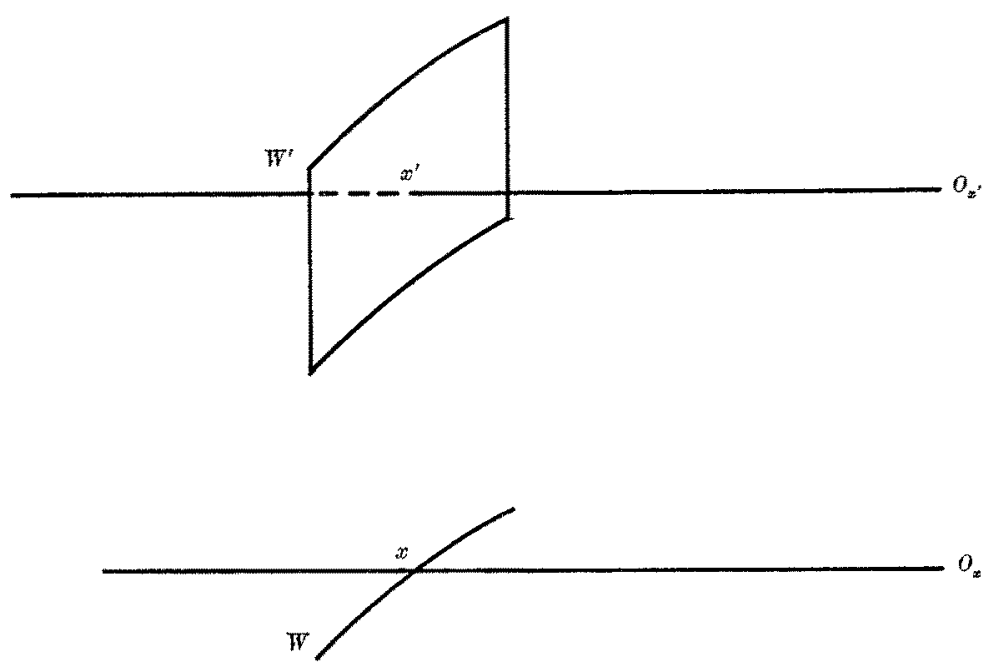
Remarque 20 . - Soit $\Phi$ un groupoïde de base $B$, il existe une action naturelle de $\Phi$ sur $B$ : celle définie par l'application $(u, x) \rightarrow \beta(u)$ de $\underset{\alpha=1}{\# B} \operatorname{sur} B$.

Il est possible d'interpréter toutes les actions d'un groupoide de cette façon. En effet, si $\Phi$ opère sur la variété $E$, les applications suivantes définissent sur $\Phi_{\alpha=p}^{\not \# E}$



$$
\begin{aligned}
& \alpha(u, x)=x ; \quad \beta(u, x)=u x \\
& \mu[(u, x),(v, u x)]=(u v, x) \\
& \nu(u, x)=\left(u^{-1}, u x\right) .
\end{aligned}
$$

Muni de cette structure, $\Phi_{\alpha=\infty}^{\#} E$ est le groupoüde des éléments composables pour l'action de $\Phi$ sur $E$. Il est immédiat que les orbites, pour l'action naturelle de $\Phi_{\alpha=p}^{\# E}$ sur sa base $E$, sont les mêmes que les orbites pour l'action de $\Phi$ sur $E$.

\section{C) Formes canoniques.}

Soient $E$ une variété, $T E$ le fibré tangent à $E$ et $I T$ la projection de $T E$ sur $E$. Soit $\Phi$ un groupoïde différentiable et soient $\delta$ et $\delta^{\prime}$ des actions de $\Phi$ sur $E$ et $T E$ compatibles avec $\Pi$. Soit enfin $p$ la projection de $E$ sur la base $B$ de $\Phi$.

Définition 21. - On appelle forme canonique associée aux actions de $\Phi$ sur $E$

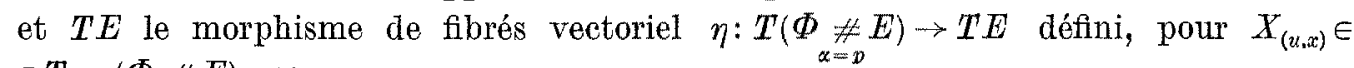
$\in T_{(u, x)}\left(\Phi_{\alpha=p}^{\# E), \text { par : }}\right.$

$$
\eta\left(X_{(u, x)}\right)=\delta^{\prime}\left(u^{-1}, T \delta\left(X_{(u, x)}\right)\right)
$$

EXEMPLE 22. - Soit $\Phi$ un groupoïde de base $B$ opérant sur $T B$; pour $X_{u} \in T_{u} \Phi$, on a alors:

$$
\eta\left(X_{u}\right)=u^{-1} T \beta\left(X_{u}\right)
$$

On convient, plus généralement, étant donnée une sous-variété $M$ de $\underset{\alpha=y}{\#} \underset{\alpha}{\#}$ d'appeler forme canonique induite sur $M$ par les actions $\delta$ et $\delta^{\prime}$ de $\Phi$ sur $E$ et $T E$ la restriction de $\eta$ au fibré $T M$. Ce morphisme est alors à valeurs dans le fibré $\grave{T} N$ induit sur $N=p(M)$ par $T E$.

\section{I. - Geometrie des sous-variétés d'une variété $\Gamma$-structurée.}

\section{A)}

Soient $G$ un groupe de Lie, $\theta: T G \rightarrow T_{\theta} G$ sa forme de structure, $M$ une variété connexe, $u_{1}$ et $u_{2}$ des applications différentiables de $M$ dans $G$. Il est bien connu 
qu'une condition nécessaire et suffisante pour que $u_{2}=\gamma \circ u_{i}$, avec $\gamma$ translation à gauche de $G$, est que $u_{2}^{*} \theta=u_{1}^{*} \theta$. Ce paragraphe a pour but le démonstration d'un résultat analogue lorsque $G$ est le groupoïde de définition d'un pseudogroupe de Lie de type fini.

\section{§1. La forme canonique sur $\Pi^{r+1}(V)$.}

Soit $V$ une variété de classe $C^{\infty}$. Pour tout entier $r=0,1, \ldots$, on adopte les notations suivantes:

1) $\Pi^{r}(V)$ est la variété des jets d'ordre r des difféomorphismes locaux de $V$,

2) $\stackrel{\circ}{I}^{r}(V)$ est la variété des jets d'ordre $r$ de l'application identique de $V$.

On identifie $\check{I}^{r}(V)$ à $V$ par le difféomorphisme $x \rightarrow j_{x}^{r} 1$

3) $\alpha$ (resp. $\beta$ ), l'application source (resp. but) est définie par:

$$
\alpha\left(j_{x}^{r} \varphi\right)=x \quad\left(\operatorname{resp} \cdot \beta\left(j_{x}^{r} \varphi\right)=\varphi(x)\right)
$$

4) $\mu: \Pi^{r}(V) \underset{\beta=\alpha}{\not \#} \Pi^{r}(V) \rightarrow \Pi^{r}(V)$ est définie par la composition des jets:

$$
\mu\left(j_{w}^{\tau} \varphi, j_{y}^{*} \psi\right)=j_{x}^{\tau}(\psi \circ \varphi)
$$

5) $\nu: \Pi^{r}(V) \rightarrow \Pi^{r}(V)$ est définie par $v\left(j_{x}^{r} \varphi\right)=j_{\varphi(x)}^{r} \varphi^{-1}$.

On note encore $\Pi^{r}(V)$ le groupoïde différentiable défini par les données précédentes.

Définition 1.1. - Soit $\varphi$ un difféomorphisme local de $V$, de source $U^{\prime}$ et de but $U^{\prime \prime}$. On appelle relèvement de $\varphi$ à $\Pi^{r}(V)$ et on note $\varphi^{r}$ le difféomorphisme local de $I^{r}(V)$ de source $\beta^{-1}\left(U^{\prime}\right)$ et de but $\beta^{-1}\left(U^{\prime \prime}\right)$ défini par:

$$
\varphi^{r}\left(j_{x}^{r} \psi\right)=j_{.0}^{*}(\varphi \circ \psi)
$$

Le résultat suivant est bien connu:

Proposition 1.2. - Il existe pour tout $r$ un plongement canonique de $\Pi^{r+1}(V)$ dans $\Pi \Pi^{\wedge}\left[\Pi^{r}(\nabla)\right]$.

Soit en effet $A \in \Pi^{r+1}(V)$ et $\varphi$ un difféomorphisme local de $V$ tel que $A=j_{a s}^{r+1} \varphi$. Soit 1 l'application identique de $V$, le relèvement $\varphi^{r}$ de $\varphi$ à $\Pi^{r}(V)$ est défini au voisinage de $j_{x}^{r} 1$ et le prolongement $T_{\left(j_{x}^{r} 1\right)} \varphi^{r}$ de $\varphi^{r}$ aux vecteurs tangents à $\Pi^{r}(V)$ d'origine $j_{s}^{r} 1$ ne dépend que de $A$ (et non de $\varphi$ ). L'application qui à $A=j_{x}^{r+1} \varphi$ fait correspondre $T_{\left(j_{x}^{r} 1\right)} \varphi^{r} \in \Pi^{1}\left[\Pi^{r}(V)\right]$ est le prolongement annoncé.

Le groupoïde $\Pi^{\top}\left[\Pi^{r}(V)\right]$ opère naturellement sur sa base $\Pi^{r}(V)$ et sur l'espace tangent $T^{\prime}\left(\Pi^{r}(V)\right)$ et ces deux actions sont compatibles avec la projection de $T\left(\Pi^{r}(V)\right)$ 
sur $\Pi^{r}(V)$. On a donc, sur $\Pi^{\mathrm{1}}\left(\Pi^{r}(V)\right) \underset{\alpha=1}{\#} \Pi^{r}(V)$ une forme canonique, qu'on notera $\eta$.

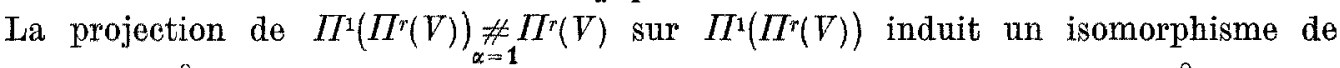
$\Pi^{r+1}(V) \# \stackrel{\circ}{\Pi}^{r}(V)$ sur $\Pi^{r+1}(V)$ et done la forme canonique sur $\Pi^{r+1}(V) \# \stackrel{\circ}{\Pi}^{r}(V)$ définit un morphisme $\theta^{x+1}$ de $T\left(\Pi^{r+1}(V)\right)$ sur le fibré $T\left(\Pi^{r}(V)\right)$, induit par $T\left(\Pi^{r}(V)\right)$ sur $\Pi^{r}(V)$. On dit que $\theta^{r+1}$ est la forme canonique sur $\Pi^{r+1}(V)$.

Soit $\alpha_{r+1}$ (resp. $\beta_{r+1}$ ) l'application de $\Pi^{r+1}(V)$ dans $\Pi^{r}(V)$ (resp. $\Pi^{r}(V)$ ) définie par: $\theta_{r+1}\left(j_{x}^{r+1} \varphi\right)=j_{x}^{r} 1\left(\operatorname{resp} . \beta_{r+1}\left(j_{x}^{r+1} \varphi\right)=j_{x}^{r} \varphi\right) ; \alpha_{r+1}\left(\right.$ resp. $\left.\beta_{r+1}\right)$ est la restriction a $\Pi^{r+1}(V)$ de l'application source (resp. but) de $\Pi^{1}\left(\Pi^{r}(V)\right)$ sur $\Pi^{r}(V)$ et l'application $\Pi_{r+1}=$

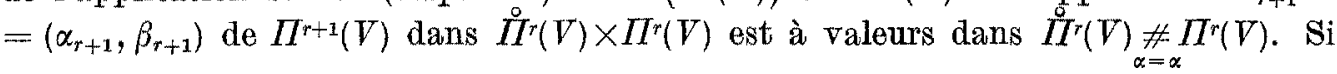
$A=j_{x}^{r+1} \varphi \in \Pi^{r+1}(V)$ et si $X \in T_{A} \Pi^{r+1}(V)$ est un vecteur tangent en $A$ a $\Pi^{r+1}(V)$, on a done:

$$
\theta^{r+1}(X)=\left[T_{\alpha_{r+1}(A)} \varphi^{r}\right]^{-1}\left[T \beta_{r+1}(X)\right]
$$

L'intérêt de la forme $\theta^{r+1}$ provient du résultat suivant qui est l'analogue pour les groupoïdes d'un résultat obtenu en [6] dans le cadre des espaces fibrés:

THÉonìme 1.3. - Soit $f$ un difféomorphisme local de $\Pi^{r+1}(V)$. Une condition nécessaire et suffisante pour qu'au voisinage de chaque point de sa source $f$ coïncide avec le relèvement à $\Pi^{r+1}(V)$ d'un difféomorphisme local de $V$ (i.e. $f=\varphi^{r+1}$ pour un certain $\varphi$ ) est que $f^{*} \theta^{r+1}=\theta^{r+1}$.

Preuve. - La condition est évidemment nécessaire, montrons qu'elle est suffisante. Puisque $f^{*} \theta^{r+1}=\theta^{r+1}$, on a d'une part, $\alpha_{r+1}=f \circ \theta_{r+1}$ et d'autre part, $T \beta_{r+1}(X)=$ $=0 \Rightarrow T \beta_{r+1} \circ T f(X)=0 ; f$ laisse donc globalement invariantes les $\alpha_{r+1}$-fibres de $\Pi^{r+1}(V)$ et, localement, une $\beta_{r+1}$-fibre de $\Pi^{r+1}(V)$ est transformée par $f$ en une $\beta_{r+1-}$ fibre. La projection $\Pi_{r+1}=\left(\alpha_{r+1}, \beta_{r+1}\right)$ de $\Pi^{r+1}(V)$ sur $\grave{\Pi}^{r}(V) \not \prod_{\alpha=\alpha}(V)$ étant une submersion, il existe done localement un difféomorphisme $\tilde{f}_{1}$ de $\prod_{\substack{\alpha \\ \Gamma}}(V) \underset{\alpha=\alpha}{\not} \prod^{r}(V)$ sur luimême, de la forme $(a, b) \stackrel{\vec{f}_{2}}{\rightarrow}\left(a, f_{1}(b)\right)$, avec $f_{1}$ difféomorphisme local de $I^{r}(V)$, tel que:

$$
\Pi_{r+1} \circ f=\tilde{f}_{1} \circ \Pi_{r+1} .
$$

Il en résulte que:

$$
\beta_{r+1} \circ f=f_{1} \circ \beta_{r+1}
$$

et

$$
f_{1}^{*} \theta^{r}=\theta^{r} \quad\left(\theta^{r} \text { forme canonique sur } \Pi^{r}(V)\right)
$$

Montrons que $f$ est la restriction à $\Pi^{r+1}(V)$ du relèvement $f_{1}^{1}$ de $f_{1} \grave{a} \Pi^{1}\left[\Pi^{r}(V)\right]$. Soit $A \in \Pi^{r+1}(V)$, on pose $a=\alpha_{r+1}(A)$ et $b=\beta_{r+1}(A)$; comme $f(A)$ et $f_{1}^{1}(A)$ sont des isomorphismes de $T_{a}\left[\Pi^{r}(V)\right]$ sur $T_{f_{1}(b)}\left[\Pi^{r}(V)\right]$, on aura $f(A)=f_{1}^{1}(A)$ si pour tout $X \in T_{a}\left[\Pi^{r}(V)\right]$, on a $f(A)(X)=f_{1}^{1}(A)(X)$. Or, soit $Y \in T_{A}\left[\Pi^{r+1}(V)\right]$ tel que $A(X)=$ 
$=T \beta_{r+1}(Y)$, on a:

$$
f_{1}^{1}(A)(X)=T f_{1} \circ A(X)=T f_{1} \circ T \beta_{r+1}(Y)=T \beta_{r+1} \circ T f(Y)
$$

et

$$
[f(A)]^{-1} \circ T \beta_{r+1} \circ T f(Y)=\theta^{r+1} \circ T f(Y)=\theta^{r+1}(Y)=A^{-1} \circ A(X)=X
$$

done $f(A)(X)=f_{1}^{1}(A)(X)$ d'où $f(A)=f_{1}^{1}(A)$.

Le même raisonnement appliqué à $f_{1}$ permet d'obtenir un difféomorphisme local $\left(f_{1}\right)_{1}=f_{2}$ de $\Pi^{r-1}(V)$ tel que $f_{1}=f_{2}^{1} \mid \Pi^{r}(V)$ (restriction de $f_{2}^{1}$ à $\Pi^{r}(V)$ ) et

$$
f_{2}^{*} \theta^{r-1}=\theta^{r-1},
$$

et ainsi de suite; on construit de proche en proche pour $i \leqslant r+1$ un difféomorphisme local $f_{i}$ de $\Pi^{r+1-i}(V)$ tel que $f_{i-1}=f_{i}^{i} \mid \Pi^{r+2-i}(V)$. Le difféomorphisme $f_{r+1}$ est un difféomorphisme local de $V \times V$ de la forme

$$
(x, y) \rightarrow(x, \varphi(y))
$$

avec $\varphi$ difféomorphisme de $V$; on vérifie aisément que localement $f=\varphi^{r+1}$.

§2. La forme de structure d'un pseudo-groupe de Lie de type fini.

Soit $\Gamma$ un pseudo-groupe de difféomorphismes locaux de $V[7]$, pour tout $r=0,1, \ldots$

$$
\Phi^{r}=\left\{j_{x}^{r} \varphi / \varphi \in \Gamma, x \in \text { source de } \varphi\right\},
$$

est un sous-groupoïde de $\Pi^{r}(\nabla)$ et la restriction de $\beta_{r}$ à $\Phi^{r}$ applique $\Phi^{r}$ sur $\Phi^{r-1}$. Une solution de $\Phi^{r}$ est un difféomorphisme local $\varphi$ de $V$ tel que, pour tout $x$ appartenant à la source de $\varphi$ on ait: $j_{x}^{r} \varphi \in \Phi^{r}$. On dit que $\Gamma$ est complet d'ordre $q$ si $\Gamma$ est l'ensemble des solutions de $\Phi^{q}$. Si $\Gamma$ est complet d'ordre $q$, il est alors complet d'ordre $s>q$.

Définition 2.1. - On dit qu'un pseudo-groupe $\Gamma$ est un pseudo-groupe de Lie de type fini si les conditions suivantes sont satisfaites:

1) Pour tout $r$. le groupoïde associé $\Phi^{r}$ est une sous-variété de $\Pi^{r}(V)$.

2) Il existe $q$ tel que $\Gamma$ soit complet d'ordre $q$.

3) Il existe $s$ tel que la restriction de $\beta_{s}$ à $\Phi^{s}$ soit localement un difféomorphisme $\operatorname{sur} \Phi^{s-1}$.

Le plus petit entier $q$ (resp. $s$ ) pour lequel la condition 2 (resp. 3) est satisfaite est l'ordre (resp. le degré) de $\Gamma$. Le degré est supérieur ou égal à l'ordre. 


\section{Remarques 2.2 .}

1) On n'impose pas à un pseudo-groupe de Lie de type fini $\Gamma$ d'opérer transivement sur la variété $V$.

2) Pour tout $r$, $\Phi^{r}$ est un sons-groupoïde différentiable de $\Pi^{r}(V)$.

Exemple 2.3. - Soit $O^{n}$ le groupe orthogonal à $n$ variables. Ce groupe opère naturellement d'une part sur $\boldsymbol{R}^{n}$ et d'autre part sur l'ouvert $\boldsymbol{R}^{n}-\{0\}$ de $\boldsymbol{R}^{n}$. Désignons par $\Gamma_{1}$ (resp. $\Gamma_{2}$ ) le pseudo-groupe déduit de l'action de $\mathrm{O}_{n}$ sur $\boldsymbol{R}^{n}$ (resp. $\left.\boldsymbol{R}^{n}-\{0\}\right)$. Le pseudo-groupe $\Gamma_{1}$ n'est pas un pseudo-groupe de Lie de type fini car $\Phi^{0}$, qui est un cône dans $\Pi_{0}\left(\boldsymbol{R}^{n}\right)=\boldsymbol{R}^{n} \times \boldsymbol{R}^{n}$, n'est pas une sous-variété de $\Pi^{0}\left(\boldsymbol{R}^{n}\right)$. Par contre, $\Gamma_{2}$ est un pseudo-groupe de lie type fini, d'ordre 1 et de degré 2 .

Convenons de noter $j_{x} \varphi$ le germe de $\varphi$ en $x$ et soit:

$$
\Phi=\left\{j_{x} \varphi / \varphi \in \Gamma, x \in \text { source de } \varphi\right\} .
$$

La loi de décomposition $\left(j_{\psi(x)} \varphi\right) \cdot\left(j_{x} \psi\right)=j_{x}(\varphi \circ \psi)$ munit $\Phi$ d'une structure de groupoïde de base $V$. Pour tout $r$, l'application $j^{r}$ de $\Phi$ sur $\Phi^{r}$ définie par:

est un homomorphisme.

$$
j^{r}\left(j_{x} \varphi\right)=j_{x}^{r} \varphi,
$$

Proposimion 2.4. - Soit $\Gamma$ un pseudo-groupe de Lie de type fini de degré $s_{0}$. Pour $\varepsilon \geqslant s_{0}, j^{s}$ est un isomorphisme de $\Phi$ sur $\Phi^{s}$.

Soit $s \geqslant s_{0}$ et soient $\varphi_{1}$ et $\varphi_{2} \in \Gamma$ tels que $j_{x_{0}}^{s} \varphi_{1}=j_{x_{0}}^{s} \varphi_{2}$; les applications $j^{s-1} \varphi_{1}$ et $j^{s-1} \varphi_{2}$ de $V$ dans $\Phi^{s-1}$ ont même application linéaire tangente en $x_{0}$. Comme $\Phi$ est localement difféomorphe à $\Phi^{s-1}$, les applications $j^{s} \varphi_{1}$ et $j^{s} \varphi_{2}$ ont aussi la même propriété. Done, en associant à: $A=j_{x_{\mathrm{s}}}^{s} \varphi_{1}$, l'espace tangent en $A$ à l'image de $j^{s} \varphi_{1}$, on obtient sur $\Phi^{s}$ un champ d'éléments de contact complètement intégrable dont les variétés intégrales sont les images des applications $j^{s} \varphi$ pour $\varphi \in T$. Il en résulte que si $j_{x}^{s} \varphi_{1}=j_{x}^{s} \varphi_{2}$, on a $j_{x} \varphi_{1}=j_{x} \varphi_{2}$ d'où le résultat.

Corollatre 2.5. - Pour $s>s_{0}, \beta_{s}$ induit un difféomorphisme de $\Phi^{s}$ sur $\Phi^{s-1}$.

Il suffit en effet de remarquer que $\beta_{s}=\left(j^{s-1}\right) \circ\left(j^{s}\right)^{-1}$.

Soit $A \in \Phi s_{0}$, notons encore $A$ l'unique élément de $\Phi^{s_{0}+1}$ dont l'image par $\beta_{s_{0}+1}$ est précisément $A$. Considéré comme élément de $\Pi^{1}\left[\Pi^{s_{0}}(V)\right]$ (cf. proposition 1.2), $A$ induit un isomorphisme de $T_{\alpha(A)} \Phi^{s_{0}}$ sur $T_{A} \Phi^{s_{0}}$; son inverse, qu'on notere $A^{-1}$, applique done $T_{A} \Phi^{s_{\mathrm{o}}}$ sur $T_{\alpha(A)} \Phi^{s_{\mathrm{o}}}$. Soit alors, $\stackrel{\circ}{T}^{s_{\mathrm{n}}}$ le fibré induit par $T \Phi^{\mathrm{s}}$ sur la variété $I^{s_{s}}(V)$ des unités de $\Phi^{s_{s}}$.

DÉfINITIon 2.6. - On appelle forme de structure du pseudo-groupe $T$ et on note $\omega$ le morphisme de fibrés vectoriels de $T \Phi^{s_{0}}$ sur ${ }^{T} \Phi^{s_{0}}$ compatible avec l'application

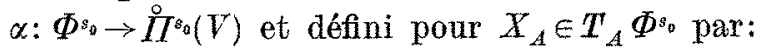

$$
\omega_{A}\left(X_{A}\right)=A^{-1}\left(X_{A}\right) .
$$


Soit $\varphi \in \Gamma$, pour tout $r$, le relèvement $\varphi^{r}$ de $\varphi$ à $\Pi^{r}(V)$ induit un difféomorphisme local de $\Phi^{r}$, qu'on note encore $\varphi^{r}$ et qu'on appelle relèvement de $\varphi$ à $\Phi^{r}$.

ThÉork̀me 2.7. - Soit $f$ un difféomorphisme local de $\Phi^{s_{0}}$. Une condition nécessaire et suffisante pour qu'au voisinage de chaque point de sa source $f$ coïncide avec le relèvement à $\Phi^{s_{0}}$ d'un difféomorphisme $\varphi \in \Gamma$ est que $f^{*} \omega=\omega$.

Preuve. - Comme $\beta_{s_{0}}$ est localement un difféomorphisme, son prolongement aux vecteurs, $T \beta_{s_{0}}$, induit un isomorphisme de $\stackrel{\circ}{T} \Phi^{s_{0}}$ sur $\stackrel{\mathscr{T}}{T} \Phi^{s_{0}-1}$. Si l'on note encore $\theta^{s_{0}}$ la restriction à $T \Phi^{s_{0}}$ de la forme canonique de $\Pi^{s_{0}}(\nabla)$, il est immédiat que $\theta^{s_{0}}$ est à valeurs dans $\stackrel{i}{T} \Phi^{s_{0}-1}$ et que:

$$
T \beta_{s_{0}} \circ \omega=\theta^{s_{0}} .
$$

On a donc: $f^{*} \omega=\omega \Leftrightarrow f^{*} \theta^{s_{0}}=\theta^{s_{0}}$.

La nécessité de la condition est alors immédiate. Pour montrer que la condition est suffisante, on remarque d'abord, par un raisonnement analogue à celui de la proposition 1.2, que, pour tout $r, \Phi^{r+1}$ se plonge canoniquement dans $\Pi^{1}\left[\Phi^{r}\right]$. Ceci étant, on procède comme pour la démonstration du théorème $1.4: f$ définit un difféomorphisme local $f_{1}$ de $\Phi^{s_{0}-1}$ dont le relèvement $f_{1}^{1} \grave{a} \Pi^{1}\left[\Phi^{s_{0}-1}\right]$ coïncide avec $f$ en restriction à $\Phi^{s_{a}}$, de même $f_{1}$ définit $f_{2}$ difféomorphisme local de $\Phi^{s_{0}-\mathbf{z}}$ et ainsi de suite jusqu'à obtenir un difféomorphisme local $\varphi$ de $V$ dont les relèvements successifs $\varphi^{r}$ induisent les difféomorphismes locaux $f_{r}$ de $\Phi^{s_{0}-r}$ pour $r \leqslant s_{0}$ et tel que $f=\varphi^{s_{0}}$. Ce difféomorphisme appartient alors à $\Gamma$ car pour tout $x$ appartenant à la source de $\varphi, j_{x}^{s_{0}} \varphi=$ $=\varphi^{s_{0}}\left(j_{x}^{s_{3}} 1\right)=f\left(j_{x}^{s_{0}} 1\right)$ appartient à $\Phi^{s_{\mathrm{s}}}$ et done $\varphi$ est solution de $\Phi^{s_{\mathrm{v}}}$ d'où le résultat.

On pose désormais $\Phi^{s_{0}}=\Phi$; on dit que $\Phi$ est le groupoïde de définition de $\Gamma$.

ThÉORÈme 2.8. - Soient $u_{1}$ et $u_{2}$ deux applications différentiables d'une variété dans $\Phi$. Une condition nécessaire et suffisante pour que localement on ait:

est que

$$
u_{2}=\varphi \circ u_{1} \quad \text { avec } \varphi \in \Gamma
$$

$$
u_{1}^{*} \omega=u_{2}^{*} \omega
$$

Preuve. - La condition est évidemment nécessaire. Elle est aussi suffisante. Soient en effet $\Pi_{1}$ et $\Pi_{2}$ les première et seconde projections de $\Phi \times \Phi$ sur $\Phi$ et soit $\Phi \underset{\alpha=\alpha}{\# \Phi}$ la sous-variété de $\Phi \times \Phi$ formés des couples $(A, B)$ tels que $\alpha(A)=\alpha(B)$. Puisque $u_{1}^{*} \omega=u_{2}^{*} \omega$, l'application $u: x \rightarrow\left(u_{1}(x), u_{2}(x)\right)$ de $M$ dans $\Phi \times \Phi$ est à valeurs dans le produit fibré $\Phi_{\alpha=\alpha}^{\# \Phi} \Phi$ et de plus, si on pose $\Omega=\Pi_{1}^{*} \omega-\Pi_{2}^{*} \omega$, on a $u^{*} \Omega=0$. Soit $C$ le champ déléments de contact défini sur $\Phi \times \Phi$ par $\Omega=0$. La restriction de $\omega$ à $T_{A} \Phi$ étant un isomorphisme pour tout $A$, la dimension du champ $O$ est égale à celle de $\Phi$.

Soit $(A, B) \in \Phi \underset{\alpha=\alpha}{\#} \Phi$ et soient $\varphi_{1}$ et $\varphi_{2} \in \Gamma$ tels que $A=j_{x}^{s_{s}} \varphi_{1}$ et $B=j_{x}^{s_{s}} \varphi_{2}$; le relèvement de $\varphi=\varphi_{2} \circ \varphi_{1}^{-1} \grave{a} \phi$, qu'on notera encore $\varphi$, vérifie $\varphi^{*} \omega=\omega$ (théorème 2.6). 
Le graphe de $\varphi$ est done une variété intégrale de dimension maximale du champ $C$, entièrement contenue dans $\underset{\alpha=\alpha}{\#} \Phi$, et passant par $(A, B)$. La restriction $\tilde{C}$ du champ $C$ à $\Phi \underset{\alpha=\alpha}{\# \Phi}$ est donc un champ complètement intégrable dont les variétés intégrales ont mêmes germes que les graphes des relèvements à $\Phi$ des difféomorphismes de $\Gamma$. Comme $u$ est à valeurs dans $\underset{\alpha=\alpha}{\#} \Phi$ et vérifie $u^{*} \Omega=0$, $u$ est nécessairement à valeurs dans une variété intégrale de $\tilde{C}$ (en restreignant éventuellement la source de $u$ ), d'où le résultat.

\section{B)}

Lorsqu'un groupe de Lie $G$ opère sur une variété $V$ on sait, dans les cas réguliers, depuis les travaux de $\mathrm{S}$. Lie et $\mathrm{E}$. Cartan entre autres, ramener le problème de l'égalité modulo $G$ de deux germes de sous-variétés à un problème de comparaison d'invariants différentiels. On démontre un résultat analogue en remplaçant les groupes par des pseudo-groupes de Lie de type fini. Le $\S 1$ a pour but de fixer un certain nombre de notations; le $\$ 2$ contient les résultats.

§1. Les espaces $\mathfrak{P}^{\lambda} ; \overline{\mathfrak{T}}^{\lambda} ; P^{r} ; C P^{r} ; \mathfrak{T}^{\lambda . r}$ associés à une variété $V$ de classe $C^{\infty}$.

a) Germes d'immersions et germes de sous-variétés. - Soit $\mu$ une immersion de $\boldsymbol{R}^{p}$ dans $V$ et $j_{x} \mu$ le germe de $\mu$ en $x$; on pose $\alpha\left(j_{x} \mu\right)=x$ et $\beta\left(j_{x} \mu\right)=\mu(x)$. L'ensemble $\mathfrak{f}^{\lambda}$ des germes d'immersions de $\boldsymbol{R}^{p}$ dans $V$ est un faisceau de base $\boldsymbol{R}^{p}$, il est donc, en tant qu'espace étalé, naturellement muni d'une structure de variété de dimension $p$ pour laquelle $\alpha$ est un difféomorphisme au voisinage de tout point. Au voisinage de $j_{x} \mu$, on $a: \beta=\mu \circ \alpha$ et done $\beta$ est une immersion.

On note $\overline{\mathfrak{T}}^{\lambda}$ le quotient de $\mathfrak{T}^{\lambda}$ par la relation: "il existe un difféomorphisme local $\sigma$ de $\boldsymbol{R}^{s}$ tel que $j_{x} \mu=j_{x}\left(\nu_{\circ} \sigma\right) "$ ". $\bar{\jmath}^{\lambda}$ est la variété des germes de sous-varietés de dimension $p$ de $V$. L'application $\beta$ passe au quotient et induit une application $\bar{\beta}$ de $\overline{\mathfrak{T}}^{2}$ sur $V$ qui est aussi une immersion. Etant donnée une immersion $\mu$ de $\boldsymbol{R}^{p}$ dans $V$, on convient de noter $\bar{j}_{x} \mu$ le germe de sous-variété défini par $j_{\alpha} \mu \in \mathfrak{T}^{\lambda}$.

Soit $\varphi$ (resp. $\sigma$ ) un difféomorphisme local de $V$ (resp. $\boldsymbol{R}^{p}$ ), le prolongement de $\varphi$ (resp. $\sigma$ ) à $\mathfrak{T}^{\lambda}$ est le difféomorphisme local $\varphi_{p}$ (resp. $\sigma_{p}$ ) de $\mathfrak{T}^{\lambda}$ défini par $\varphi_{p}\left(j_{x} \mu\right)=$ $=j^{\lambda}(\varphi \circ \mu)\left(\right.$ resp. $\left.\sigma_{p}\left(j_{x} \mu\right)=j_{\sigma-1_{i x}}(\mu \circ \sigma)\right)$. On $a \alpha \circ \varphi_{p}=\alpha\left(\operatorname{resp} . \beta \circ \sigma_{p}=\beta\right)$ et done $\varphi_{p}$ (resp. $\sigma_{p}$ ) est un $\alpha$-difféomorphisme (resp. $\beta$-difféomorphisme).

On définit le prolongement $\bar{\varphi}_{p}$ de $\varphi \grave{a} \bar{T}^{2}$ par passage de $\varphi_{p}$ au quotient:

$$
\bar{\varphi}_{p}\left(\bar{j}_{x} \mu\right)=\bar{j}_{x}(\varphi \circ \mu) \text {. }
$$

b) Repères et étéments de contact d'ordre $\%$ - Un repère d'ordre $r$ et de dimension $p$ de $V$ est le jet d'ordre $r$, de source 0 , d'une immersion de $\boldsymbol{R}^{p}$ dans $V$ définie au voisinage de 0 . Il exis te sur l'espace $P^{r}$ des repère d'ordre $r$ et de dimension $p$ de $V$ une 
structure canonique d'espace fibré différentiable de base $V$ dont la projection est l'application but de $\boldsymbol{P}^{r}$ sur $V$.

Le groupe $L_{p}^{r}$ des $r$-jets inversibles de $\boldsymbol{R}^{p}$ dans $\boldsymbol{R}^{p}$ de source et but 0 opère dans $P^{r}$ : si $l=j_{0}^{r} \sigma \in L_{p}^{r}$ et si $a=j_{0}^{r} \mu \in P^{r}$, alors $l \cdot a=j_{0}^{r}(\mu \circ \sigma)$. On note $C P^{r}$ l'espace des orbites de $P^{r}$ pour cette action; les éléments de $C P^{r}$ sont les éléments de contact de dimension $p$ et d'ordre $r$ de $V$. On note $I I$ la projection de $P^{r}$ sur $C P^{r}$ et l'on convient, pour $j_{0}^{r} \mu \in P^{r}$ de poser $\Pi\left(j_{0}^{r} \mu\right)=\bar{j}_{0}^{r} \mu$. L'application de $C P^{r}$ sur $V$ obtenue par passage de $\beta: P^{r} \rightarrow V$ au quotient est notée $\tilde{\beta}:$ pour $X \in C P^{r}, \tilde{\beta}(X)$ est l'origine de $X$.

Il existe sur $C P^{r}$ une structure de variété (nécessairement unique!) telle que $\Pi$, soit une submersion. De plus, la projection $\tilde{\beta}$ est une fibration différentiable de $C P^{r}$ dont la fibre est la variété des éléments de contact d'ordre $r$ de dimension $p$ et d'origine $O$ dans $\boldsymbol{R}^{n}(n=\operatorname{dim} V)$.

Soit $\varphi$ un difféomorphisme local de $V$, le prolongement $\varphi^{r}\left(\right.$ resp. $\left.\varphi^{r, e}\right)$ de $\varphi$ à $P^{r}$ (resp. $\left.C P^{r}\right)$ est le difféomorphisme local de $P^{r}\left(\right.$ resp. $\left.C P^{r}\right)$ défini par: $\varphi^{r}\left(j_{0}^{r} p\right)=j\left({ }_{0}^{r} \varphi \circ \mu\right)$ (resp. $\varphi^{r, a}\left(\vec{j}_{0}^{r} \mu\right)=\bar{j}_{0}^{r}(\varphi \circ \mu)$ ).

Pour $x \in \boldsymbol{R}^{p}$, soit $\tau_{x}$ la translation de $\boldsymbol{R}^{p}$ définie par $\tau_{x}(0)=x$. On définit une application $\beta_{r}$ de $\mathfrak{T}^{\lambda}$ dans $P^{r}$ par: $\beta_{r}\left(j_{x} \mu\right)=j_{0}^{r}\left(\mu \circ \tau_{x}\right)$. Au voisinage de $j_{x} \mu \in \mathfrak{T}^{\lambda}, \beta_{r}$ est composée de $\alpha: \mathfrak{T}^{\lambda} \rightarrow \boldsymbol{R}^{p}$ et de l'application $x \rightarrow j_{0}^{r}\left(\mu \circ \tau_{x}\right)$; ces deux applications étant des immersions, $\beta_{r}$ en est aussi une.

On note $\mathfrak{f}^{\lambda . r}$ la variété des germes d'immersions de $\boldsymbol{R}^{p}$ dans $P^{r}$. Soit $\mu: \boldsymbol{R}^{p} \rightarrow V$ une immersion, et soit $j^{r} \mu: x \rightarrow \beta_{r}\left(j_{x} \mu\right)$ le relèvement de $\mu$ dans $\boldsymbol{P}^{r}$. L'application $j^{r} \mu$ est une immersion de $\boldsymbol{R}^{p}$ dans $P^{r}$ comme composée de deux immersions. On définit une application $j^{r}$ de $\mathfrak{T}^{\lambda}$ dans $\mathfrak{j}^{\lambda \cdot r}$ par: $j^{r}\left(j_{x} \mu\right)=j_{x}\left(j^{r} \mu\right)$ et cette application, qui vérifie $\beta \circ j^{r}=\beta_{r}$ est une immersion car $\beta$ et $\beta_{r}$ le sont.

c) Actions de $\Phi$ sur $\mathfrak{T}^{2}, \overline{\mathfrak{T}}^{\lambda}, P^{r}, C P^{r}$. - Soit $\Gamma$ un pseudo-groupe de type fini sur $V$ et soit $\Phi$ le groupoïde de définition de $\Gamma$ (ef. I, A); on a des actions différentiables de $\Phi$ sur $\mathfrak{T}^{\lambda}, \bar{\top}^{2}, P^{r}$ et $C P^{r}$ compatibles avec les projections canoniques de ces variétés sur $V$. Plus précisément:

L'action de $\Phi$ sur $T^{2}$ est définie par:

$$
\delta\left(j_{x} \varphi, j_{x} \mu\right)=\varphi_{p}\left(j_{x} \mu\right) .
$$

L'action de $\Phi$ sur $\overline{\mathfrak{T}}^{\lambda}$ est définie par:

$$
\delta\left(j_{x} \varphi, \bar{j}_{x} \mu\right)=\bar{\varphi}_{p}\left(j_{x} \mu\right) .
$$

L'action de $\Phi$ sur $P^{r}$ est définie par:

$$
\delta\left(j_{x} \varphi, j_{0}^{r} \mu\right)=j_{0}^{r}(\varphi \circ \mu) .
$$

L'action de $\Phi$ sur $C P^{r}$ est définie par:

$$
\delta\left(j_{x} \varphi, \bar{j}_{0}^{r} \mu\right)=\varphi^{r, e}\left(\bar{j}_{0}^{r} \mu\right) .
$$


On remarquera en outre que ces différentes actions sont compatibles entre elles et que la condition $\mathrm{A}$ (cf. 0 ) est vérifiée.

\section{§2. Egalité, modulo $\Gamma$, des germes de sous-variétes.}

Dans ce qui suit, $\Gamma$ est un pseudo-groupe de Lie de type fini de degré $s_{0}$ sur $V$; $\Phi$ est le groupoïde de définition de $\Gamma$.

Soient $\mu$ et $v$ deux immersions de $\boldsymbol{R}^{o}$ dans $V$ définies au voisinage de $x$.

Défintaions 2.1. - On dit que les germes d'immersions $j_{x} \mu$ et $j_{x} \nu$ sont égaux modulo $\Gamma$, s'il existe une transformation $\varphi \in \Gamma$ telle que:

$$
j_{x}(\varphi \circ \mu)=j_{x} \nu
$$

On dit que les germes de sous-variétés $\bar{j}_{x} \mu$ et $\bar{j}_{x} \nu$ sont égaux modulo $\Gamma$, s'il existe un difféomorphisme local $\sigma$ de $\boldsymbol{R}^{p}$ tel que les germes d'immersions $j_{x} \mu$ et $j_{x}(\boldsymbol{v} \circ \sigma)$ soient égaux modulo $\Gamma$.

Soit $\Gamma_{p}\left(\right.$ resp. $\bar{\Gamma}_{g}$ ) le pseudo-groupe engendré dans $\mathscr{T}^{\lambda}$ (resp. $\overline{\mathfrak{T}}^{\lambda}$ ) par les prolongements à $\mathscr{T}^{\lambda}$ (resp. $\overline{\mathfrak{T}}^{\lambda}$ ) des difféomorphismes de $\Gamma$. Avee les notations $d u$ $\S 1, a$ ) il est immédiat que deux éléments $\mathcal{A}$ et $\mathscr{B} \in \mathscr{T}^{\lambda}$ (resp. $\overline{\mathcal{A}}$ et $\overline{\mathcal{B}} \in \mathfrak{T}^{2}$ ) sont égaux modulo $\Gamma$, s'ils appartiennent à la même classe d'intransitivité de $\Gamma_{p}$ (resp. $\bar{\Gamma}_{p}$ ) dans $\mathfrak{T}^{\lambda}$ (resp. $\overline{\mathfrak{T}}^{\lambda}$ ) ou, ce qui est équivalent, à la même orbite pour l'action de $\Phi$ dans $\mathscr{T}^{\lambda}$ (resp. $\overline{\mathfrak{T}}^{\lambda}$ ) définie au $\S 1$.

Pour $s \geqslant r$, la projection de $P^{s}$ sur $P^{r}$ qui à $j_{0}^{s} \mu$ associe $j_{0}^{r} \mu$ est une submersion et les actions $d u$ groupoïde $\Phi$ sur $P^{s}$ et $P^{r}$ sont compatibles avec cette projection. On peut done appliquer le corollaire 19 du chapitre 0 et done, si $U \subset P^{r}$ est un ouvert décomposable, son image réciproque $U^{\prime} \subset P^{s}$ est décomposable. De plus, si $w$ est une sous-variété adaptée à $U$, son image réciproque $W^{\prime}$ dans $P^{s}$ est adaptée à $U^{\prime}$. Ceci permet donc d'associer à la décomposition $\left(\Pi_{1}, \Pi_{2}\right): U \rightarrow \Phi \times W$ de $U$ une décomposition $\left(\Pi_{1}^{\prime}, \Pi_{2}^{\prime}\right): U^{\prime} \rightarrow \Phi \times W^{\prime}$ de $U^{\prime}$.

Définition 2.2. - Un ouvert $u \subset \mathfrak{T}^{\lambda}$ est décomposable d'ordre $r$ si $U$ est l'image réciproque par $\beta_{r}: \mathfrak{T}^{\lambda} \rightarrow P^{r}$ d'un ouvert décomposable $U$ dans $P^{r}$ et si $r$ est le plus petit entier ayant cette propriété.

Soit $\mathcal{U}=\beta_{r}^{-1}(U)$ un ouvert décomposable d'ordre $r$ dans $\mathfrak{T}^{\lambda}$ et soit $\left(\Pi_{1}, \Pi_{2}\right): U \rightarrow$ $\rightarrow \Phi \times W$ une décomposition de $U$. On définit pour chaque $s \geqslant r$ une application, notée $\left(\Pi_{1}^{s}, \Pi_{2}^{s}\right)$, de $\mathcal{U}$ dans $\Phi \times W^{\prime}$ en composant la projections $\beta_{s}$ de $U$ sur $U^{\prime} \subset P^{s}$ avec la décomposition $\left(\Pi_{1}^{\prime}, \Pi_{2}^{\prime}\right): U^{\prime} \rightarrow \Phi \times W^{\prime}$ associée à $\left(\Pi_{1}, \Pi_{2}\right)$. On dira que l'application $\left(\Pi_{1}^{s}, \Pi_{2}^{s}\right)$ est la décomposition d'ordre $s$ de $U$ associée à $\left(\Pi_{1}, \Pi_{2}\right)$.

THÉonìve 2.3. - Soit $\mathcal{U}=\beta_{r}^{-1}(U)$ un ouvert décomposable d'ordre $r$ dans $\mathfrak{T}^{\lambda}$, soit $\left(\Pi_{1}, \Pi_{2}\right)$ une décomposition de $U$ et soit $\left(\Pi_{1}^{r+1}, \Pi_{2}^{r+1}\right)$ la décomposition d'ordre $r+1$ de $\mathcal{U}$ associée à $\left(\Pi_{1}, \Pi_{2}\right)$. Pour qu'un $\alpha$-difféomorphisme local $f$ de $U$ (i.e. 
$\alpha=\alpha \circ f)$ appartienne au pseudogroupe $\Gamma_{p}$, il faut et il suffit que l'on ait:

$$
\Pi_{2}^{r+1} \circ f=\Pi_{2}^{r+1}
$$

Prevye. - Le condition est évidemment nécessaire, montrons qu'elle est suffisante. Soit $\mathcal{A}$ un élément de la source de $f$, soit $B=f(\mathcal{A})$ et soient $\mu$ et $\nu$ des immersions définies au voisinage de $x_{0}$ dans $\boldsymbol{R}^{p}$ telles que ot $=j_{x_{0}} \mu$ et $B=j_{x_{0}} \nu$. Soit $U^{\prime}$ l'image réciproque de $U$ dans $P^{r+1}$, la condition (1) implique que pour tout $x$ voisin de $x_{0}, j_{x}^{r+1} \mu$ et $j_{x}^{r+1} v$ appartiennent à la même orbite de $\Phi$ dans $U^{\prime}$. Dans $U$, $j_{x}^{r} \mu$ et $j_{x}^{*} \nu$ appartiennent done aussi à la même orbite et de plus, il existe $\varphi_{x} \in \Gamma$ tel que, en $x$, les applications linéaires tangentes de $j^{r} v$ et $\varphi_{x}^{r} \circ j^{r} \mu$ soient égales. Si done on pose $u_{1}=\Pi_{1} \circ j^{r} v$ et $u_{2}=\Pi_{1} \circ j^{r} \mu$, on $a$, pour tout $x$ voisin de $x_{0}: T_{x} u_{2}=$ $=T_{x}\left(p_{x} \circ u_{1}\right)$. Comme la forme de structure $\omega$ sur $\Phi$ est invariante par $\Gamma$, on a done $u_{1}^{*}(\omega)=u_{2}^{*}(\omega)$ d'où par le théorème $2.8 \mathrm{du} \mathrm{A}$ ), l'existence de $\varphi \in \Gamma$ tel que $u_{2}=\varphi \circ u_{1}$. En projetant par $\beta: \Phi \rightarrow V$, cette derniére égalité dans $V$, on en déduit que les applications $f$ et $\varphi_{p}$ coïncident au voisinage de $f t$ d'où le résultat.

Convenons, étant donné $j_{x} \mu \in \mathfrak{T}^{\lambda}$, de noter $\tilde{\mu}$ la section canonique de $\alpha: \mathfrak{P}^{\lambda} \rightarrow \boldsymbol{R}^{p}$ telle que $\tilde{\mu}(x)=j_{x} \mu$. Avec les notations du théorème précédent, on a:

Corollatre 2.4. - Pour que les éléments $\mathcal{A}=j_{x} \mu$ et $\mathfrak{B}=j_{x} v$ de $U$ soient égaux modulo $\Gamma$ il faut et il suffit qu'au voisinage de $x$ on ait:

$$
\Pi_{2}^{r+1} \circ \tilde{\mu}=\Pi_{2}^{r+1} \circ \tilde{\nu}
$$

Il suffit en effet de remarquer que la condition (2) équivaut à l'existence d'un $\alpha$-difféomorphisme $f\left(=\tilde{v} \circ \tilde{\mu}^{-1}\right)$ de $U$ tel que $f(\mathcal{A})=\mathfrak{B}$ et $\Pi_{2}^{r+1} \circ f=\Pi_{2}^{r+1}$.

Le passage des germes d'immersions aux germes de sous-variétés se fait sans difficulté. On énonce uniquement les résultats, laissant les démonstrations au lecteur.

Définimox 2.5. - Un ouvert $\overline{\boldsymbol{U}} \subset \overline{\mathfrak{T}}^{\lambda}$ est décomposable d'ordre $r$ si $\overline{\mathrm{U}}$ est l'image réciproque par $\bar{\beta}_{r}: \overline{\mathcal{T}}^{\lambda} \rightarrow C P^{r}$ d'un ouvert décomposable $\bar{U}$ dans $C P^{r}$, avec $r$ minimum.

Proposition 2.6. - Si $\bar{U}$ es un ouvert décomposable dans $C P^{r}$, alors les images réciproques $U^{\prime}$ et $U$ de $\bar{U}$ dans $O P^{r+1}$ et $P^{r}$, sont décomposables.

Corollatre 2.7. - Si $\bar{\tau} \subset \overline{\mathfrak{T}}^{2}$ est décomposable d'ordre $r$, son image réciproque

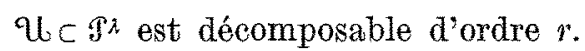

On en déduit le:

THÉork̀Me 2.8. - Soit $\overline{\boldsymbol{U}} \subset \overline{\mathfrak{J}}^{\lambda}$ un ouvert décomposable d'ordre $r, \mathcal{U} \subset \mathfrak{P}^{\lambda}$ l'image réciproque de $\overline{\mathrm{U}}$ dans $P^{2}$ et $\left(\Pi_{1}^{r+1}, \Pi_{2}^{r+1}\right)$ me décomposition d'ordre $r+1$ de $u$ associée à une décomposition $\left(I_{1}, \Pi_{2}\right)$ de $U=\beta_{r}(U)$. Une condition nécessaire et suffisante pour que $\bar{j}_{x} \mu$ et $\bar{j}_{y} v$ soient égaux modulo $T$ est qu'il existe un difféomorphisme local $\sigma$ de $\boldsymbol{R}^{p}$ tel que $\sigma(x)=y$ et:

$$
\Pi_{2}^{s+1} \circ \tilde{\mu}=\Pi_{2}^{r+1} \circ(\tilde{\nu} \circ \tilde{\sigma}) .
$$


Ce qui précède montre que, pour les ouverts décomposables d'un certain ordre dans $\overline{\mathfrak{T}}^{\lambda}$, le problème de l'égalité modulo $\Gamma$ de deux germes de sous-variétés appartenant à ces ouverts, est une fois donnée une décomposition d'un ordre convenable, ramené à un problème classique d'analyse: la recherche, étant donnée deux applications $v$ et $w$, d'un difféomorphisme $\sigma$ tel que $w=v \circ \sigma$. Il reste done à indiquer un procédé permettant de déterminer explicitement les ouverts décomposables de $\overline{\mathfrak{T}}^{\lambda}$ ainsi que des décompositions de ces ouverts à un certain ordre. Il suffit pour cela (cf. proposition 2.6) de savoir déterminer, dans $C P^{r}$, les ouverts décomposables et des décompositions de ces ouverts. C'est précisément à la recherche de ces ouverts dans $C P^{r}$ qu'est adaptée la méthode du repère mobile de $\mathrm{E}$. Cartan que l'on étudie en II.

\section{II. - La méthode du repère mobile.}

La méthode $\mathrm{du}$ repère mobile dont le but est, comme nous venons de le dire, la recherche des ouverts décomposables dans les espaces $C P^{r}(r=1,2, \ldots)$ est une méthode récurrente. Pour en démonter le mécanisme, il suffit donc, et c'est ce que nous ferons, d'indiquer de façon précise le passage de l'ordre 0 à l'ordre 1 . Comme précédemment, $\Phi$ désigne le groupoïde de définition d'un pseudo-groupe de Lie de type fini $\Gamma$ sur $V$. Les actions de $\Phi$ sont celles définies au (I, B, $\S 1)$.

\section{§. Invariants différentiels et semi-invariants.}

Définition 1.1. - Soit $U$ un ouvert de $C P^{r}$ saturé pour l'action de $\Phi$ dans $C P^{r}$. On dit qu'une fonction numérique différentiable $f: U \rightarrow \boldsymbol{R}$ est un invariant différentiel si, la restriction de $f$ à chaque orbite de $\Phi$ dans $U$ est constante.

Il est bien évident que si $f: U \rightarrow \boldsymbol{R}^{n}$ est une $\Phi$-submersion (cf. 0 ) chacune des composantes $f_{1}, \ldots, f_{n}$ de $f$ est un invariant différentiel sur $U$. Réciproquement, la connaissance au voisinage de tout point de $C P^{r}$ des invariants différentiels permet de construire des $\Phi$-submersions et done, de déterminer les points réguliers et les ouverts décomposables. Ce qui suit est précisément consacré à la détermination des invariants différentiels.

On suppose donnée une $\Phi$-submersion $f_{0}$ definie sur un ouvert saturé $U_{0}$ de $V$ et $\grave{a}$ valeurs dans un espace qu'on peut toujours supposer être un espace $\boldsymbol{R}^{\text {t }}$.

Soit $U_{1}$ l'image réciproque de $U_{0}$ dans $C P^{1}$ par la projection canonique de $C P^{1}$ dans $V$. On se propose d'indiquer comment ramener la recherche des invariants différentiels dans $U_{1}$ à la résolution d'un système différentiel défini sur un espace convenable. 
On fait choix d'une sous-variété $N_{0}$ de $V$ image d'une section de $f_{0}$. Ce choix permet de définir un certain nombre d'espaces dont les liens sont résumés dans le diagramme ci-dessous:



Les flèches horizontales (resp. verticales) non nommées sont des injections (resp. projections) canoniques.

$\Phi_{N_{0}} \quad$ groupoïde d'isotropie de $\Phi$ au-dessus de $N_{0}$ est le sous-groupoïde de $\Phi$ formé des $X \in \Phi$ tels que $\alpha(X)$ soit égal à $\beta(X)$ et appartienne à $N_{0}$. C'est une somme de groupes de Lie.

$\Phi \# N_{0} \quad$ est le produit fibré de $\Phi$ et $N_{0}$ pour les applications $\alpha: \Phi \rightarrow V$ et $i$, injection canonique de $N_{0}$ dans $V$.

$\Phi_{N_{0}} \# N_{0} \quad$ est le produit fibré de $\Phi_{N_{0}}$ et $N_{0}$ pour les applications $\alpha$ et $i$, e'est une sous-variété de $\Phi \# N_{0}$, isomorphe à $\Phi_{N_{6}}$.

$\Delta_{0} \quad$ est l'ensemble des éléments de $\Phi_{N_{0}} \# N_{0}$ de la forme $\left(j_{x} 1, x\right)$. On peut l'identifier à l'ensemble des unités de $\Phi_{N_{0}}$.

$\psi^{0} \quad$ est définie par: $\psi^{0}\left(j_{x} \varphi, x\right)=\varphi(x)=\beta\left(j_{x} \varphi\right)$.

$\psi_{0}^{0} \quad$ est la restriction de $\psi^{0}$ à $\Phi_{N_{0}} \not \# N_{0}$. C'est en fait la projection sur $N_{0}$ :

$N_{0}^{1} \quad$ est la sous-variété de $C P^{1}$ formée des éléments de contact dont l'origine appartient à $N_{0}$. C'est un fibré de base $N_{0}$. 
$\Phi \# N_{0}^{1} \quad$ est le produit fibré de $\Phi$ et $N_{0}^{1}$ pour les applications $\alpha: \Phi \rightarrow V$ et $\tilde{\beta}: N_{0}^{1} \rightarrow V$, où $\tilde{\beta}$ est la restriction à $N_{0}^{1}$ de la projection canonique $\tilde{\beta}$ de $C P^{1}$ sur $V$.

$\Delta_{0}^{1} \quad$ est l'ensemble des éléments de $\Phi_{N_{0}} \# N_{0}^{1}$ de la forme $\left(j_{x} 1, x^{1}\right)$. C'est done l'ensemble des unités de $\Phi_{N_{0}} \# N_{0}^{1}$ pour la structure de groupoïde, somme de groupes de Lie, de base $N_{0}^{1}$.

$\psi^{1} \quad$ est définie par: $\psi^{1}\left(j_{x} \varphi, x^{1}\right)=\varphi^{1,0}\left(x^{1}\right)$.

$\psi_{0}^{1} \quad$ est la restriction de $\psi^{1}$ à $\Phi_{N_{0}} \# N_{0}^{1}$.

$\stackrel{\circ}{T}^{\prime}\left(\Phi_{N_{0}} \# N_{0}^{1}\right) \quad$ est une somme d'algèbres de Lie: e'est l'algébroïde de Lie de $\Phi_{N_{0}} \# N_{0}^{1}$ considéré comme groupoïde (somme de groupes de Lie) de base $N_{0}^{1}$. C'est donc l'ensemble des vecteurs tangents à $\Phi_{N_{0}} \# N_{0}^{1}$ dont l'origine appartient à $\Delta_{0}^{1}$ et qui sont verticaux pour la projection de $\Phi_{N_{0}} \not \# N_{0}^{1}$ sur $N_{0}^{1}$ qui à $\left(j_{x} \varphi, x^{1}\right)$ associe $x^{1}$.

$T\left(\Phi_{N_{0}} \# N_{0}^{1}\right) \quad$ (resp. $T N_{0}^{1}$ ) est le fibré tangent à $\Phi_{N_{0}} \not \# N_{0}^{1}$ (resp. $N_{0}^{1}$ ).

$T \psi_{0}^{1} \quad$ est le prolongement de $\psi_{0}^{1}$ aux vecteurs.

$\stackrel{\circ}{T}^{\prime} \psi_{0}^{1} \quad$ est la restriction de $T \psi_{0}^{1}$ à $\stackrel{T}{ }^{\prime}\left(\Phi_{N_{0}} \not N_{0}^{1}\right)$.

La restriction à $\Phi_{N_{0}} \# N_{0}^{1}$ de l'application de $\Phi \# U_{1}$ sur $U_{1}$ définissant l'action de $\Phi$ sur $U_{1}$ est à valeurs dans $N_{0}^{1}$ et définit une action de $\Phi_{N_{0}}$ sur $N_{0}^{1}$. Soit $\Phi_{x}$ le groupe d'isotropie de $\Phi$ en $x \in N_{0}$ et soit $\left(N_{0}^{1}\right)_{x}$ le fibre da $N_{0}^{1}$ d'origine $x$, c'est-à-dire l'ensemble des éléments de contact (de dimension $p$ ) d'origine $x$; le groupe $\Phi_{x}$ opère naturellement sur $\left(N_{0}^{1}\right)_{x}$ et l'action de $\Phi_{N_{0}}$ sur $N_{0}^{1}$ est la "somme" pour $x \in N_{0}$, des actions de $\Phi_{x} \operatorname{sur}\left(N_{0}^{1}\right)_{x}$. En particulier, les orbites de $\Phi_{N_{0}}$ dans $N_{0}^{1}$ sont toutes situées dans les fibres de $N_{0}^{1}$ et se projettent done dans $N_{0}$ suivant des points par la projection de $N_{0}^{1}$ sur $N_{0}$ induite par la projection de $C P^{1}$ sur $V$. On désignera par invariants sur $N_{0}^{1}$ les fonctions numériques différentiables définies sur des ouverts saturés de $N_{0}^{1}$ (i.e. réunions d'orbites pour l'action de $\Phi_{N_{0}}$ sur $N_{0}^{1}$ ) et constantes sur les orbites de $\Phi_{N_{0}}$ dans $N_{0}^{\mathbf{1}}$.

Soit $f$ un invariant différentiel sur $U_{1}$, la restriction $f^{\prime}$ de $f$ à $N_{0}^{1}$ vérifie: $\left(T f^{\prime}\right)$ 。 $\circ\left(\stackrel{\circ}{T}^{\prime} \psi_{0}^{1}\right)=0$. Sous certaines conditions, la connaissance des solutions de cette équation permet de déterminer les invariants différentiels sur $U_{1}$. Plus précisément:

DÁFINition 1.2. - On appelle semi-invariant sur $N_{0}^{1}$ toute application différentiable numérique $l$ dénie sur un ouvert saturé de $N_{0}^{1}$ et telle que:

$$
(T l) \circ\left(\stackrel{\circ}{T}^{\prime} \psi_{0}^{1}\right)=0 .
$$

On remarquera qu'un semi-invariant est une fonction localement constante sur chaque orbite de $\Phi_{N_{0}}$ dans $N_{0}^{1}$. 
THÉonìme 1.3. - Si, pour tout $x \in U_{0}$ le groupe d'isotropie $\Phi_{x}$ est connexe, tout semi-invariant sur $N_{0}^{1}$ est la restriction d'un invariant différentiel de $U_{1}$ et d'un seul.

Le théorème est une conséquence immédiate des lemmes suivants:

LEMME 1.4. - Il existe, pour tout $x^{1} \in U_{1}$, une submersion définie au voisinage de $x^{1}$, à valeurs dans $N_{0}^{1}$ et compatible avec les relations d'équivalence définies par $\Phi$, $\operatorname{sur} U_{1}$ et $\Phi_{N_{0}}$ sur $N_{0}^{1}$.

Soit en effet $x$ la projection de $x^{1}$ dans $U_{0}$ et soit $\varepsilon$ une section différentiable de $\psi^{r}$ définie sur un voisinage $\mathcal{U}_{x}$ de $x$ (une telle section existe car $\psi_{0}$ est une submersion $\left(0\right.$, théorème 1.6)). Soit $\Pi_{1}$ la projection de $\Phi \# N_{0}$ dans $\Phi$ et soit $\varepsilon_{1}=\Pi_{1} \circ \varepsilon$; si $\mathcal{u}_{x^{1}}=\tilde{\beta}-1\left(\mathcal{u}_{x}\right)$ est l'image réciproque de $\mathcal{U}_{x}$ par la projection $U_{1} \rightarrow U_{0}$, l'application $S_{\varepsilon}$ définie sur $\mathcal{u}_{x^{1}}$ par: $\Phi_{\varepsilon}\left(y^{1}\right)=\left[\varepsilon_{1}\left(\tilde{\beta}\left(y^{1}\right)\right)\right]^{-1}\left(y^{1}\right)$ est à valeurs dans $N_{0}^{1}$ et vérifie les conditions annoncées.

Lemme 1.5. - Tout invariant différentiel sur $N_{0}^{1}$ (pour l'action de $\Phi_{N_{0}}$ ) se prolonge en un invariant différentiel sur $U_{1}$.

Soit en effet $h$ un invariant défini sur un ouvert $\checkmark$ de $N_{0}^{1}$. Le saturé $\tilde{V}$ de $v$ dans $U_{1}$ est ouvert car, avec les notations du lemme précédent, pour $x^{1} \in U_{1}, \tilde{V} \cap$ $\cap \mathcal{U}_{x^{1}}=S_{\varepsilon}^{-1}(\mathcal{U}) \cap \mathcal{U}_{x^{1}}$ est ouvert. De plus, deux sections $\varepsilon$ et $\varepsilon^{\prime}$ de $\psi^{0}$ étant données au dessus de $\mathcal{U}_{x}$, les fonctions définis sur $\tilde{\vartheta} \cap \mathcal{U}_{x^{1}}$ par $h_{\circ} S_{\varepsilon}$ et $h \circ S_{\varepsilon^{\prime}}$, sont égales car $S_{\varepsilon}$ et $S_{\varepsilon^{\prime}}$, sont compatibles avec les relations d'équivalence sur $U_{1}$ et $N_{0}^{1}$. On définit done, sur $\widetilde{\mathcal{V}}$, une fonction $\tilde{h}$ par: $\tilde{h} \mid \widetilde{\mathcal{O}} \cap \mathcal{U}_{x^{1}}=h \circ S_{\varepsilon}=h \circ S_{\varepsilon^{\prime}}$, et $h$ est un invariant sur $\widetilde{v}$.

LEMmE 1.6. - Si les groupes d'isotropies $\Phi_{x}$ sont connexes pour tout $x \in U_{0}$, alors, sur $N_{0}^{1}$, tout invariant différentiel est un semi-invariant et réciproquement.

En effet, pour $x^{1} \in N_{0}^{1}$, d'origine $x \in N_{0}$, l'espace tangent à l'orbite de $x^{1}$ dans $N_{0}^{1}$ est l'image par $\stackrel{T}{T}^{\prime} \psi_{0}^{1}$ de la fibre de $\mathscr{T}^{\prime \prime}\left(\Phi_{N_{0}} \not N_{0}^{1}\right)$ au-dessus de $x^{1}$ et done, un invariant est toujours un semi-invariant. Si les groupes d'isotropie de $\Phi$ sont connexes sur $U_{0}$, pour tout $x_{1} \in N_{0}^{1}$, l'orbite de $x^{1}$ est connexe; un semi-invariant étant une fonction localement constante sur chaque orbite, $y$ est done alors constante, d'où le résultat.

REMaRque 1.7. - Soit $G$ un groupe de Lie et $H$ un sous-groupe fermé de $G$. Si $H$ n'est pas connexe, les groupes d'isotropie pour l'action naturelle de $G$ sur $G / H$ no sont pas connexes. Soit $H_{0}$ la composante connexe de l'identité dans $H$; pour l'action naturelle de $G$ sur $G / H_{0}$ les groupes d'isotropie sont connexes. Comme la projection canonique de $G / H_{0}$ sur $G / H$ est un revêment. tout germe d'immersion dans $G / H_{0}$ se projette dans $G / H$ en un germe d'immersion. On en déduit done une application de $\mathfrak{P}^{\lambda}\left(G / H_{0}\right)$ (variété des germes d'immersion de $\boldsymbol{R}^{p}$ dans $\left.G / H_{0}\right)$ sur $\mathfrak{P}^{2}(G / H)$ (variété des germes d'immersion de $\boldsymbol{R}^{p}$ dans $G / H$ ). De plus, les actions de $G$ sur $G / H_{0}$ et $G / H$, étant compatibles avec la projection de $G / H_{0}$ sur $G / H$, la projection de $\mathfrak{S}^{\lambda}\left(G / H_{0}\right)$ sur $\mathfrak{T}^{\lambda}\left(G^{\lambda} H\right)$ est compatible avec les relations d'équivalence définies par $G$ sur $\mathfrak{T}^{\lambda}\left(G / H_{0}\right)$ et $\mathfrak{T}^{\lambda}(G / H)$. Au lieu d'étudier l'action de $G$ sur $G / H$, il suffit done détudier celle de $G$ 
sur $G / H_{0}$. On appelle orientation des éléments de $\mathfrak{P}^{2}(G / H)$ (resp. $\overline{\mathfrak{T}}^{\lambda}(G / H)$ l'opération qui consiste a passer de $\mathfrak{f}^{\lambda}(G / H)$ ) (resp. $\overline{\mathfrak{T}}^{\lambda}(G / H)$ à $\mathfrak{T}^{\lambda}\left(G / H_{0}\right)$ (resp. $\overline{\mathfrak{T}}^{\lambda}\left(G / H_{0}\right)$ ). Son intérêt est de permettre d'utiliser le théorème 1.3 pour la recherche des invariants différentiels. Cette technique s'étend parfois (au moins localement) au cas où e'est un groupoïde $\Phi$ qui opère sur une variété $E$. On doit alors considérer le groupoïde d'isotropie $\Psi$ de $\Phi$ et le sous-groupoïde $\Psi_{0}$ de $\Psi$ dont les fibres sont les composantes connexes des unités des fibres de $\Psi$ et s'assuser que $\Phi / \Psi_{0}$ a une structure de variété telle que l'action de $\Phi$ sur $\Phi / \Psi_{0}$ soit différentiable et que la projection de $\Phi / \Psi=E$ soit un revêtement. Ceci une fois lait, il suffiit d'étudier l'action de $\Phi$ sur $\Phi / \Psi_{0}$, action pour laquelle les groupes d'isotropie sont connexes.

\section{B)}

Les notations sont celles du paragraphe précédent. On suppose désormais que les groupes d'isotropie de $\Phi$ sur $U_{0}$ sont connexes. La détermination des invariants différentiels sur $U_{1}$ se ramène alors à celle des semi-invariants dans $N_{0}^{1}$, e'est-à-dire à la résolution du système différentiel $(T l) \circ\left(\stackrel{T}{ }^{\prime} \psi_{0}^{1}\right)=0$ sur $N_{0}^{1}$ (avec $l$, application différentiable de $N_{0}^{1}$ dans $\boldsymbol{R}$, comme inconnue). Pour résoudre effectivement ce système, on doit l'écrire explicitement. Ceci est a priori difficile, car la source et le but de $\mathscr{T}^{\prime \prime} \psi_{0}^{1}$ ne possèdent pas de coordonnées reliées simplement à celles de $U_{0}$. La méthode du repère mobile permet cette écriture explicite sans utiliser l'expression de $\psi_{1}$ en coordonnées, nous le montrons au $\$ 1$. On étudie au $\$ 2$ le passage de l'ordre o à l'ordre 1.

\section{§1. La règle fondamentale.}

a) Les trivialisations locales adaptées a $T^{\prime} N_{0}^{1}$. - Rappelons d'abord comment, à une base $\varepsilon_{1}, \ldots, \varepsilon_{n}$ d'un espace vectoriel $E$, est associé un atlas $\left(O_{i}, \varphi_{i}\right)_{i \varepsilon I}$ de la grasmannienne $G_{p}(E)$ des sous-espaces vectoriels de dimension $p$ de $E$.

Soit $i$ une permutation de $\{1, \ldots, n\}$ dont les restrictions à $\{1, \ldots, p\}$ et $\{p+1$, $\ldots, n\}$ sont croissantes. Soit $E_{i}^{\prime}$ (resp. $E_{i}^{\prime \prime}$ ) le sous-espace engendré par $\varepsilon_{i_{1}}, \ldots, \varepsilon_{i_{p}}$ (resp. $\left.\varepsilon_{i_{p+1}}, \ldots, \varepsilon_{i_{n}}\right)$; on a $E=E_{i}^{\prime} \oplus E_{i}^{\prime \prime}$ et on désigne par $\Pi_{i}^{\prime}$ (resp. $\left.\Pi_{i}^{\prime \prime}\right)$, la projection de $E$ sur $E_{i}^{\prime}$ (resp. $\left.E_{i}^{u}\right)$. L'ouvert $O_{i}$ de $G_{p}(E)$ est l'ensemble des sous-espaces vectoriels de dimension $p$ de $E$ qui se projettent $\operatorname{sur} E_{i}^{\prime}$ par $\Pi_{i}^{\prime}$. Pour $F \in O_{i}, \varphi_{i}(F)$ est la matrice dans les bases $\varepsilon_{i_{1}}, \ldots, \varepsilon_{i_{\bar{x}}}$ et $\varepsilon_{i_{p_{n+1}}}, \ldots, \varepsilon_{i_{n}}$ de l'application linéaire de $E_{i}^{\prime}$ dans $E_{i}^{\prime \prime}$ admettant $F$ comme graphe.

Soit e une section différentiable de $N_{\theta}$ dans l'espace $H(V)$ des repères de $V$; pour $x \in N_{0}, e(x)$ est done une base $e_{1}(x), \ldots, e_{u}(x)$ de $T_{x} V$. Comme la fibre $\left(N_{0}^{1}\right)_{x}$ de $N_{0}^{1}$ au-dessus de $x$ est la grassmannienne $G_{p}\left(T_{x} V\right)$, pour chaque $x \in N_{0}$, la donnés de $e(x)$ permet, comme ci-dessus, de définir un atlas $\left(O_{i, x}, \varphi_{i, x}\right)$ dans $\left(N_{0}^{1}\right)_{x}$.

La réunion $O_{i}=\bigcup_{x \in N_{0}} O_{i, x}$ est un ouvert de $N_{0}^{1}$ et l'application $\varphi_{i}$ de $O_{i}$ sur $N_{0} \times \boldsymbol{R}^{p(n-v)}$ 
dont la restriction à $O_{i, x}$ coÏncide pour tout $x \in N_{0}$ avec $\varphi_{i, x}$ est un difféomorphisme compatible avec les projections de $O_{i}$ et $N_{0} \times \boldsymbol{R}^{p(n-p)}$ sur $N_{0}$. Soit $\mathbb{I}$ la projection de $T\left(N_{0} \times \boldsymbol{R}^{p(n-p)}\right)$ sur $\boldsymbol{R}^{p(n-p)}$ obtenue en composant la projection de $T\left(N_{0} \times \boldsymbol{R}^{p(n-p)}\right)=$ $=\left(T N_{0}\right) \times\left(T \boldsymbol{R}^{p(n-p)}\right)$ sur le second facteur avec la forme invariante à gauche $T \boldsymbol{R}^{p(n-p)} \rightarrow T_{0} \boldsymbol{R}^{y(n-p)}=\boldsymbol{R}^{p(n-p)}$, on note $\varphi_{i}^{\mathrm{h}}$ l'application de $T O_{i}$ sur $\boldsymbol{R}^{p(n-p)}$ définie par:



Soit alors, $T^{\prime} N_{0}^{1}$ le fibré des vecteurs tangents à $N_{0}^{1}$ et qui sont verticanx pour la projection de $N_{0}^{1}$ sur $N_{0}$ et soit $T^{\prime} O_{i}$ l'ensemble des vecteurs de $T^{\prime} N_{0}^{1}$ dont l'origine appartient à $O_{i}$.

LEMrue 1.1. - L'application $\tilde{\varphi}_{i}$ de $T^{\prime} O_{i}$ sur $O_{i} \times \boldsymbol{R}^{p(n-p)}$ définie pour $X_{x^{1}} \in T^{\prime} O_{i}$, vecteur d'origine $x^{1}$ dans $T^{\prime} O_{i}$, par: $\tilde{\varphi}_{i}\left(X_{x^{1}}\right)=\left(x^{1}, \varphi_{i}^{\mathbb{I}}\left(X_{x^{1}}\right)\right)$ est une trivialisation locale de $T^{\prime} N_{0}^{1}$ au-dessus de $O_{i}$.

DÉMONSTRATION. - Immédiate à partir des définitions.

DÉFINiTion 1.2. - On appelle trivialisation locale adaptée à $T^{\prime} N_{0}^{1}$ toute trivialisation du type $\tilde{\varphi}_{i}$ ci-dessus obtenue à partir d'une section différentiable e de $N_{0}$ dans $H(V)$.

Avec les mêmes notations que plus haut, soient $u_{J_{1} x}^{J_{3}}, J_{1}=1, \ldots, j ; J_{2}=1, \ldots$, $(n-p)$ les coordonnées définies par $\varphi_{i, x}$ dans $O_{i, x}$ et soient $\partial / \partial u_{J_{1} x}^{J_{2}}$ les champs de vecteurs associés à ces coordonnées sur $O_{i, x}$. Pour $\left(J_{1}, J_{2}\right)$ fixé, le champ de vecteur vecteur $\partial / \partial u_{J_{1}}^{J_{2}}$ sur $O_{i}$ dont la restriction à $O_{i, x}$ est $\partial / \partial u_{J_{1}, x}^{J_{2}}$ est une section de $O_{i}$ dans $T^{\prime} O_{i}$. Le lecteur vérifiera que la trivialisation $\tilde{\phi}_{i}$ est celle obtenue par la donnée des $p(n-p)$ sections $\partial / \partial u_{J_{1}}^{J_{2}}, J_{1}=1, \ldots, p ; J_{2}=1, \ldots,(n-p)$, de $T^{\prime} O_{i}$.

Considérons maintenant l'applieation $\mathscr{T}^{\prime} \psi_{0}^{1}$ de $\mathscr{T}^{\prime}\left(\Phi_{N_{0}} \# O_{i}\right)$ dans $T N_{0}^{1}$. Cette application est compatible avec la projection de $\Phi_{N_{0}} \# N_{0}^{1}$ sur $N_{0}^{1}$ et est à valeurs dans $T^{\prime} N_{0}^{1}$. L'ensemble $\stackrel{Q}{T}^{\prime}\left(\Phi_{N_{0}} \not O_{i}\right)$ des éléments de $i^{\prime}\left(\Phi_{N_{0}} \not N_{0}^{1}\right)$ dont l'origine appartient à $\Phi_{N_{0}} \# O_{i}$ est done appliqué sur $T^{\prime} O_{i}$ per $T^{\prime} \psi_{0}^{1}$. De plus, une fois connu $T^{\prime} \psi_{0}^{1}$ sur chacun des $T^{\prime}\left(\Phi_{N_{0}} \not O_{i}\right), T^{\prime} \psi_{0}^{1}$ est entièrement déterminée.

Donnons-nous une trivialisation locale $\tilde{\varphi}_{i}$ adaptée à $T^{\prime \prime} N_{0}^{1}$ (définition 1.2) et soient $\partial / \partial u_{J_{1}}^{J_{2}}$ les sections de $O_{i}$ dans $T^{\prime} O_{i}$ associées à $\tilde{\varphi}_{i}$ et définies ci-dessus. La restriction de $\mathscr{T}^{\prime} \psi_{0}^{\mathbf{1}}$ à $\mathscr{T}^{\prime}\left(\Phi_{N_{0}} \# O_{\dot{i}}\right)$, restriction qu'on notera encore $\stackrel{T}{T}^{\prime} \psi_{0}^{1}$, peut alors s'écrire:

$$
\stackrel{\circ}{T^{\prime}} \psi_{\theta}^{1}=\sum_{\substack{J_{1}=1, \ldots, p \\ J_{2}=1, \ldots, p-p}} \tau_{J_{1}}^{J_{z}} \otimes \frac{\partial}{\partial u_{J_{1}}^{J_{2}}}
$$

où $\tau_{J_{1}}^{J_{2}}: \stackrel{\circ}{T}^{\prime}\left(\Phi_{N_{0}} \not \# O_{i}\right) \rightarrow O_{i} \times \boldsymbol{R}$ est un morphisme de fibré. Ce sont ces morphismes que l'on va expliciter dans le paragraphe suivant.

b) Les formes de pseudo-connexions. - Soit $i^{\prime}\left(\Phi_{N_{0}} \# N_{0}\right)$ l'ensemble des vecteurs tangents à $\Phi_{N_{0}} \# N_{0}$ dont l'origine appartient à $\Delta_{0}$ et qui sont verticaux pour l'application source: $\left(j_{x} \varphi, x\right) \rightarrow x$ de $\Phi_{N_{0}} \# N_{0}$ sur $N_{0}$. Si l'on munit $\Phi_{N_{0}} \# N_{0}$ (qui 
est difféomorphe à $\left.\Phi_{N_{0}}\right)$ de la structure de groupoïde évidente, somme de groupes de Lie, de base $N_{0}$, alors $i^{\prime}\left(\Phi_{N_{0}} \# N_{0}\right)$ est l'algébroïde de Lie correspondant. En particulier, pour $x \in N_{0}$, la fibre de $\hat{T}^{\prime}\left[\Phi_{N_{0}} \# N_{0}\right)$ au dessus de $x$ s'identifie à l'algèbre de lie du groupe d'isotropie $\Phi_{x}$ de $\Phi$ en $x$.

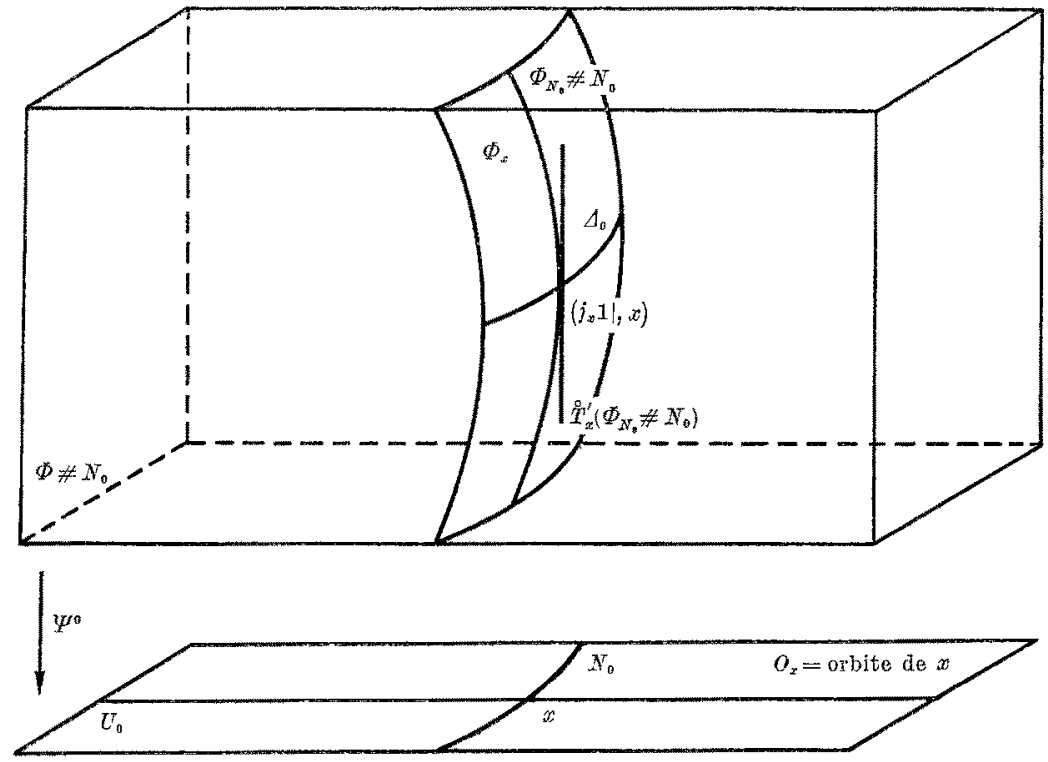

Définition 1.3. - Soit $U$ un voisinage ouvert de $\Delta_{0}$ dans $\Phi \# N_{0}$ et soit $\alpha_{U}$ la restriction à $U$ de l'application source de $\Phi \# N_{0}$ sur $N_{0}$. Une forme de pseudoconnexion sur $U$ est un morphisme de fibrés vectoriels $\omega: T U \rightarrow \Upsilon^{\prime \prime}\left(\Phi_{N_{0}} \# N_{0}\right)$ compatible avec $\alpha_{U}$ et tel que pour tout $x \in N_{0}$, le rang de la restriction de $\omega \grave{a ̀ ~} T U \cap T \Phi_{x}$ soit égal à la dimension de $\Phi_{x}$.

REMARQUE 1.4. - En pratique, les formes de pseudo-connexion qui interviennent vérifient la condition supplémentaire suivante: Pour tout $x \in N_{0}$, la restriction de $\omega$ à $T U \cap T \Phi_{x}$ coïneide avec la forme invariante à gauche canonique sur le groupe de Lie $\Phi_{x}$.

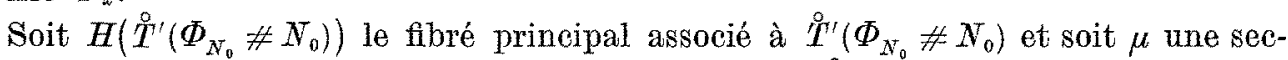
tion différentiable définie sur $N_{0}$, à valeurs dans $H\left(i^{\prime}\left(\Phi_{N_{0}} \# N_{0}\right)\right)$. (Notre étude étant locale, on peut toujours supposer qu'une telle section existe).

La donnée de $\mu$ équivaut à celle de $r^{r}$ sections différentiables $\mu_{1}, \ldots, \mu_{r}$ de $N_{0}$ dans $\stackrel{D}{T}^{\prime}\left(\Phi_{N_{0}} \# N_{0}\right)$ telles que, pour $x \in N_{0}$, les vecteurs $\mu_{1}(x), \ldots, \mu_{r}(x)$ forment une base de l'algèbre de Lie de $\Phi_{a}$.

Une forme de pseudo-connexion $\omega$ étant donnée, soit $\bar{\omega}$ le transposé de $\omega$ par la projection de $\Phi \# N_{0}^{1}$ sur $\Phi \not N_{0}$ définie par: $\left(u, x^{1}\right) \rightarrow(u, x)$ (avec $x=$ origine de $x^{1}$ ) et soit $\overline{\bar{\omega}}: T\left(\Phi \# N_{0}^{1}\right) \rightarrow \grave{T}^{\prime}\left(\Phi_{N_{0}} \# N_{0}^{1}\right)$ le morphisme obtenu en relevant $\bar{\omega}$ dans 
$\check{T}^{\prime}\left(\Phi_{N_{0}} \not \# N_{0}^{1}\right)$. Le diagramme suivant est done commutatif:

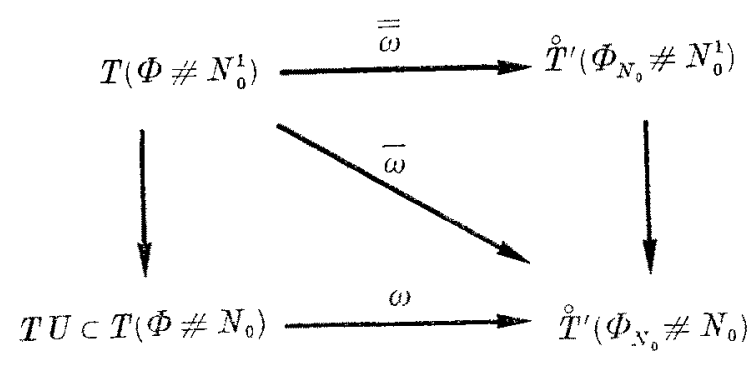

La donnée de la section $\mu$ permet alors d'écrire successivement:

$$
\omega=\sum_{k=1}^{r} \omega_{k} \otimes \mu_{k}
$$

où les $\omega_{k}$ sont des morphismes de $T U$ dans $N_{0} \times \boldsymbol{R}$ compatibles avec $\alpha_{u}: U \rightarrow N_{0}$ :

$$
\bar{\omega}=\sum_{k=1}^{r} \bar{\omega}_{k} \otimes \mu_{k},
$$

avee $\bar{\omega}_{k}$ transposé de $\omega_{k}$

$$
\overline{\bar{\omega}}=\sum_{k=1}^{r} \overline{\bar{\omega}}_{k} \otimes \mu_{k}
$$

où les $\overline{\bar{\omega}}_{k} ; T\left(\Phi \# N_{0}^{1}\right) \rightarrow N_{0}^{1} \times \boldsymbol{R}$ sont obtenus par relèvement des $\bar{\omega}_{k}$.

On note $\omega_{1}^{\prime}, \ldots, \omega_{r}^{\prime}$ les restrictions de $\overline{\bar{\omega}}_{1}, \ldots, \overline{\bar{\omega}}_{r}$ à $\grave{T}^{\prime}\left(\Phi_{N_{0}} \# N_{0}^{1}\right)$. Comme la restriction de $\omega$ à $T U \cap T \Phi_{x}$ est de rang maximal, les $\omega_{k}^{\prime}$ forment une base de l'espace des morphismes de $\stackrel{\circ}{T}^{\prime}\left(\Phi_{N_{0}} \# N_{0}^{1}\right)$ dans $N_{0}^{1} \times \boldsymbol{R}$ qui sont compatibles avec la projection de $\Phi_{N_{0}} \not \# N_{0}^{1}$ sur $N_{0}^{1}$ ou, ce qui est équivalent des morphismes $\check{T}^{\prime}\left(\Phi_{N_{0}} \not N_{0}^{1}\right) \rightarrow \boldsymbol{R}$.

Soit $\tilde{\varphi}_{i}: T^{\prime} O_{i} \rightarrow O_{i} \times \boldsymbol{R}^{p(n-p)}$ une trivialisation locale adaptée à $T^{\prime} N_{0}^{1}$ : Avec les notations du a), la restriction de $\stackrel{\circ}{ }^{\prime} \psi_{0}^{1}$ à $\stackrel{\complement}{T}^{\prime}\left(\Phi_{N_{0}} \not O_{i}\right)$ s'écrit:

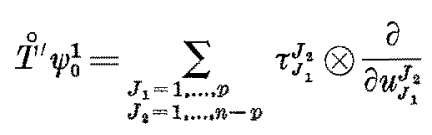

où $\tau_{J_{1}}^{J_{2}}$ est un morphisme de $\stackrel{\circ}{T}^{\prime}\left(\Phi_{N_{0}} \not O_{i}\right)$ dans $O_{i} \times \boldsymbol{R}$. On peut done écrire:

$$
\tau_{J_{1}}^{J_{2}}=\sum_{k=1}^{r} a_{J_{1} k}^{J_{2}} \omega_{k}^{\prime} J_{1}=1, \ldots, p ; J_{2}=1, \ldots, p(n-p),
$$

où les $a_{J_{1} k}^{J_{2}}$ sont des fonctions différentiables sur $O_{i}$. Ces fonctions, une fois déter- 
minées, le système différentiel fondamental

$$
(T l) \circ\left(\stackrel{T}{ }^{\prime} \psi_{0}^{1}\right)=0
$$

se ramène done sur $O_{i}$ au système suivant:

$$
\sum_{\substack{J_{1}=1, \ldots, p \\ J_{2}=1, \ldots, n-p}} \frac{\partial l}{\partial u_{J_{1}^{2}}^{J_{2}}} a_{J_{2, k}}^{J_{1}}=0 \quad k=1, \ldots, r
$$

il est done entièrement explicité. La règle de $\mathrm{E}$. Cartan (ef. d) ci-dessous) permet le calcul des fonctions $a_{J_{1} k}^{J_{3}}$.

c) La forme canonique. - Le groupoïde différentiable $\Phi$ étant le groupoïde de définition d'un pseudo-groupe de Lie de type fini $\Gamma$ sur la variété $V$, il opère naturallement sur $V$ et $T V$ et ces actions sont compatibles. Ceci permet done de définir sur $\Phi \# V$ une forme canonique (cf. 0 ). On note $\eta$ la forme canonique induite sur $\Phi \# N_{0}$.

Si $A=\left(j_{x} \varphi, x\right) \in \Phi \# N_{0}$ et si $X \in T_{A}\left(\Phi \# N_{0}\right)$ on a done:

$$
\eta(X)=\left(T_{x} \varphi\right)^{-1}\left(T \psi^{0}(X)\right) \text {. }
$$

De plus, $\eta$ est un morphisme de $T\left(\Phi \# N_{0}\right)$ sur le fibré $\mathscr{T} N_{0}$ induit par $T U_{0}$ sur $N_{0}$ (ou $U_{0}$ est l'ouvert saturé de $N_{0}$ dans $V$ ).

Proposition 1.5. - Pour toute forme de pseudo-connexion $\omega$ on $a$, au voisinage de $A_{0}$ :

$$
\operatorname{Ker} \eta \oplus \operatorname{Ker} \omega=T\left(\Phi \not N_{\theta}\right) \text {. }
$$

En effet, en un poit $\left(j_{x} 1, x\right) \in \Delta_{0}$, le noyau de $\eta$ est l'algèbre de Lie de $\Phi_{x}$ et $\omega$ est une projection sur cette algèbre de Lie, d'où l'égalité en ce point. La continuité des formes $\eta$ et $\omega$ permet de prolonger cette égalité à un voisinage de $\left(j_{x} 1, x\right)$ d'où le résultat.

Soient $e$ et $\mu$ des sections de $N_{0}$ dans $H(V)$ et $H\left(\stackrel{T}{T}^{\prime}\left(\Phi_{N_{0}} \# N_{0}\right)\right)$ respectivement, et soit $\omega$ une forme de pseudo-connexion. On a:

$$
\begin{aligned}
& \eta=\sum_{l=1}^{n} \eta_{l} \otimes e_{l}, \\
& \omega=\sum_{k=1}^{r} \omega_{k} \otimes \mu_{k},
\end{aligned}
$$

où $\eta_{t}$ et $\omega_{k}$ sont des morphismes de fibrés vectoriels de $T\left(\Phi \# N_{0}\right)$ dans $N_{0} \times \boldsymbol{R}$. En composant avec la projection de $N_{0} \times \boldsymbol{R}$ sur $\boldsymbol{R}$, chacun de ces morphismes définit une forme différentielle sur $\Phi \not N_{0}$ qu'on désignera encore par la même lettre. Comme conséquence de la proposition précédente et des définitions de $\eta$ et $\omega$, on a alors: 
Proposition 1.6. - Avee les notations ci-dessus, les formes différentielles $\eta_{l}$ et $\omega_{k}$ constituent, au voisinage de $\Delta_{0}$, une base de l'espace des formes différentielles sur $\Phi \# N_{0}$.

On suppose donnée dans ce qui suit une section différentiable e de $N_{0}$ dans $H(V)$; les notations sont celles $\mathrm{d} u a$ ). Soit $x^{\mathrm{i}} \in O_{i, x}$ et soit $M$ une sous-variété de dimension $p$ de $V$ dont l'élément de contact en $x$ est $x^{1}$. Soit $\tilde{M}=\left(\psi_{0}\right)^{-1}(M)$ l'image réciproque de $M$ dans $\Phi \# N_{0}$; on note $\tilde{\eta}$ la restriction à $\tilde{M}$ de la forme canonique $\eta$ et l'on désigne par $\tilde{\eta}_{1}, \ldots, \tilde{\eta}_{n}$ les formes différentielles induites sur $\tilde{M}$ par les formes $\eta_{1}, \ldots, \eta_{n}$ sur $\Phi \# N_{0}$.



Comme $x^{1}$ appartient à l'ouvert $O_{i, x}$ défini par $e_{i_{1}}(x), \ldots, e_{i_{p}}(x)$, les formes $\tilde{\eta}_{i_{1}}, \ldots, \tilde{\eta}_{i_{p}}$ sont indépendantes sur un voisinage $\mathcal{U}$ de $\left(j_{x} 1, x\right)$ dans $\widetilde{M}$ et, dans ce voisinage on $a$, pour $q=p+1, \ldots, n$ :

$$
\tilde{\eta}_{i q}=\sum_{s=1}^{p} t_{\alpha}^{s} \tilde{\eta}_{i_{s}}
$$

où les $t_{q}^{s}$ sont des fonctions numériques sur $\widetilde{M}$.

Si l'on convient de désigner par $\tilde{\eta}^{\prime}$ (resp. $\left.\tilde{\eta}^{\prime \prime}\right)$ la forme $\left(\tilde{\eta}_{i}:, \ldots, \tilde{\eta}_{i}\right)\left(\operatorname{resp} .\left(\tilde{\eta}_{i_{p_{1}}}, \ldots, \tilde{\eta}_{i_{n}}\right)\right)$ définie sur $\widetilde{M}$ et à valeurs dans $\boldsymbol{R}^{p}$ (resp. $\boldsymbol{R}^{n-p}$ ) l'égalité (1) s'écrit:

$$
\tilde{\eta}^{\prime \prime}=t \tilde{\eta}^{\prime}
$$

où $t$ est une fonction définie sur $\widetilde{M}$ et à valeurs dans l'espace des matrices à $p$ colonnes et $n-p$ lignes.

Soit $t^{\prime}$ la restriction de $t$ à la fibre de $\widetilde{M}$ au-dessus de $x$, c'est-à-dire à $\Phi_{x}$. Cette application $t^{\prime}$ est définie sur le voisinage ouvert $\mathcal{W}^{\prime}=\mathcal{U} \cap \Phi_{x}$ de $j_{x} 1$ dans $\Phi_{x}$. Soit 
$g \in \mathcal{U}^{\prime}$ et soit $y^{1}$ l'élément de contact de dimension $p$, de $V$ égal à $\eta\left(T_{y}(\widetilde{M})\right)$. Comme $\tilde{\eta}^{\prime}\left(T_{g}(\tilde{M})\right)$ est de dimension $p, y^{1} \in O_{i, x} ;$ de plus, relativement à la carte $\varphi_{i, x}$, la matrice des coordonnées de $y^{1}$ est la matrice $t^{\prime}(g)$ comme le montrent les égalités (1) et $\left(1^{\prime}\right)$.

Le résultat suivant est la clé de la méthode du repère mobile.

Lewre 1.7. - Pour tout $g \in \mathcal{U}^{\prime}$, on a:

$$
t^{\prime}\left(g^{-1}\right)=\varphi_{i, x}\left(g x^{1}\right)
$$

Soit en effet $g \in \mathcal{U}^{\prime}$ et $\varphi$ un difféomorphisme local, appartenant au pseudogroupe $\Gamma$, tel que $g=j_{x} \varphi$.

On $a: T_{g} \widetilde{M}=\left(T_{g} \psi_{0}\right)^{-1}\left(x^{1}\right)$ et done, si $y^{1}=\eta\left(T_{g}(\tilde{M})\right)$,

$$
\begin{aligned}
y^{1} & =\eta\left[\left(T_{g} \psi^{0}\right)^{-1}\left(x^{1}\right)\right] \\
& =\left(T_{x} \varphi^{-1}\right)\left(T_{s} \psi^{0}\right)\left(T_{g} \psi^{0}\right)^{-1}\left(x^{1}\right) \\
& =\left(T_{x} \varphi^{-1}\right)\left(x^{1}\right) \\
& =g^{-1} x^{1}
\end{aligned}
$$

mais alors $t^{\prime}(g)=\varphi_{i, x}\left(y^{1}\right)=\varphi_{i, x}\left(g^{-1} x^{1}\right)$ d'où le résultat en remplaçant $g$ par $g^{-1}$.

d) La règle de E. Cartan. - Les notations sont celles des paragraphes précédents.

On suppose données

1) Une section différentiable $e: N_{0} \rightarrow H(V)$ (cf. $a$ );

2) Une section différentiable $\mu: N_{0} \rightarrow H\left(\hat{T}^{\prime}\left(\Phi_{N_{0}} \# N_{0}\right)\right)$ (cf. $\left.b\right)$;

3) Une forme de pseudo-connexion $\omega$ sur $\Phi \# N_{0}$.

Soit $\left(O_{i}, \varphi_{i}\right)$ une carte de $N_{0}^{1}$ associée à la section $e$ (cf. $a$ ) et soit $\mathfrak{o}^{1} \in O_{i}$. Comme au $c$ ), on fait choix d'une-variété $M$ de dimension $p$ de $V$ dont l'espace tangent en $x$ est $x^{1}$ et on note $\widetilde{M}=\left(\psi^{0}\right)^{-1}(M)$.

Les formes $\tilde{\eta}_{i_{J}} ; \tilde{\eta}^{\prime} ; \tilde{\eta}^{\prime \prime}$ ont la même signification qu'au $c$ ). On note $\tilde{\omega}$ la restriction de $\omega$ à $\tilde{M}$ et on désigne par $\tilde{\omega}_{1}, \ldots, \tilde{\omega}_{r}$ les composantes de $\tilde{\omega}$ relativement à $\mu$. Comme $x^{1} \in O_{i}$, les formes $\tilde{\eta}_{i_{1}}, \ldots, \tilde{\eta}_{i_{p}}$ et $\tilde{\omega}_{1}, \ldots, \tilde{\omega}_{r}$ constituent une base de l'espace des formes différentielles sur $\widetilde{M}$ au voisinage de $j_{x} 1$. On a done, pour $q=p+1, \ldots, n$ des égalités:

$$
d \tilde{\eta}_{i q}=\sum_{l . m=1}^{p} A_{l m}^{q} \tilde{\eta}_{a k} \wedge \tilde{\eta}_{i m}+\sum_{\substack{l=1 . \ldots . p \\ k=1 \ldots . .,}} B_{l k}^{a} \tilde{\eta}_{i_{q}} \wedge \omega_{k}
$$

où $A_{l m}^{q}$ et $B_{l s}^{q}$ sont des fonctions numériques sur $\tilde{M}$. 
Avec des conventions évidentes, on écrit (1) sous la forme:

$$
d \tilde{\eta}^{\prime \prime}=A \tilde{\eta}^{\prime} \wedge \tilde{\eta}^{\prime}+B \tilde{\eta}^{\prime} \wedge \tilde{\omega}
$$

On pose $B\left(j_{x} 1\right)=B\left(x^{1}\right) ; B\left(x^{1}\right)$ peut être considéré comme la matrice d'une application linéaire de $\boldsymbol{R}^{r}$ dans $\boldsymbol{R}^{p} \times \boldsymbol{R}^{n-p}$.

On a, par ailleurs (ef. c)): $\tilde{\eta}^{\prime \prime}=t \tilde{\eta}^{\prime}$. Le champ déléments de contact défini sur $\tilde{M}$ par $\tilde{\eta}^{\prime}=\mathbf{0}$ est done le même que celui défini sur $\tilde{M}$ par $\tilde{\eta}=0$ e'est-à-dire par $T \psi_{0}=0$ Comme $\psi_{0}$ est une submersion, ce champ d'éléments de contact est complètement intégrable. Il existe donc des fonctions $\bar{A}_{l m}^{s}$ et $\bar{B}_{b k}^{s}$ sur $\widetilde{M}$ telles que, pour $s=1, \ldots, p$ :

$$
d \tilde{\eta}_{i_{s}}=\sum_{l, m=1}^{p} \bar{A}_{l m}^{s} \tilde{\eta}_{i_{2}} \wedge \tilde{\eta}_{i_{m}}+\sum_{\substack{l=1 \\ k=1 \ldots \ldots,}} \bar{B}_{l k}^{s} \tilde{\eta}_{i_{l}} \wedge \tilde{\omega}_{k} .
$$

On écrit encore (2) sous la forme:

$$
d \tilde{\eta}=\bar{A} \tilde{\eta}^{\prime} \wedge \tilde{\eta}^{\prime}+\bar{B} \tilde{\eta} \tilde{\eta}^{\prime} \wedge \tilde{\omega}
$$

On pose $\bar{B}\left(j_{x} 1\right)=\bar{B}\left(x^{1}\right)$ et on considére $\bar{B}\left(x^{1}\right)$ comme la matrice d'une application linéaire de $\boldsymbol{R}^{r}$ dans $\boldsymbol{R}^{p} \times \boldsymbol{R}^{p}$.

On peut maintenant énoncer le résultat fondamental.

Règle de E. Cartan. - Etant données, comme ci-dessus, des sections $e$ et $\mu$ et une forme de pseudo-connexion $\omega$, soit $\left(O_{i}, \varphi_{i}\right)$ une earte de $N_{0}^{1}$ associée à la section $e$ (cf. $a)$ et soient $u_{J_{1}}^{J_{2}}\left(J_{1}=1, \ldots, p ; J_{2}=1, \ldots, n-p\right)$ les coordonnées associées à $\varphi_{1}$ dans $O_{i}$. Pour tout $x^{1} \in O_{i}$, soit $t\left(x^{1}\right)=\left[t_{J_{1}}^{J_{2}}\left(x^{1}\right)\right]$ la matrice à $p$ colonnes et $n-p$ lignes des coordonnées de $x^{1}$ et soit $T\left(x^{1}\right)$ la matrice de l'application $1 \times t\left(x^{1}\right)$ de $\boldsymbol{R}^{n} \times \boldsymbol{R}^{n-y}$ dons $\boldsymbol{R}^{p} \times \boldsymbol{R}^{n-p}$.

Les expressions, dans les coordonnées $u_{J_{1}}^{J_{2}}$ des solutions du système différentiel:

$$
T l \circ \stackrel{\circ}{T}^{\prime} \psi_{0}^{1}=0
$$

dont la source est dans $O_{i}$ sont alors les solutions du système:

$$
\sum_{\substack{J_{1}=1, \ldots, p \\ J_{2}=1, \ldots, p}} \frac{\partial l}{\partial u_{y_{1}}^{J_{z}}}\left(x^{1}\right) a_{J_{1}, k}^{J_{1}}\left(x^{1}\right)=0 \quad k=1, \ldots, r
$$

où $a\left(x^{1}\right)=\left[a_{J_{1}, k}^{J_{2}}\left(x^{1}\right)\right]$, considérée comme matrice d'une aplication linéaire de $\boldsymbol{R}^{r}$ dans $\boldsymbol{R}^{p} \times \boldsymbol{R}^{n-p}$ est définie par:

$$
a\left(x^{1}\right)=B\left(x^{1}\right)-T\left(x^{1}\right) \bar{B}\left(x^{1}\right),
$$

où $B\left(x^{1}\right)$ et $\bar{B}\left(x^{1}\right)$ ont été définies plus haut.

3 - Annali di Matematica 
PreUve. - La restriction de $\grave{T}^{\prime} \psi_{0}^{1}$ a $\left(\stackrel{T}{T}^{\prime} \Phi_{N_{0}} \not \# O_{i}\right)$ peut en effet se mettre sous la forme:

$$
\stackrel{\circ}{T}^{\prime} \psi_{0}^{1}=\sum_{\substack{J_{1}=1, \ldots, p \\ J_{1}=1 \ldots . ., p-p}} \tau_{J_{1}}^{J_{2}} \otimes \frac{\partial}{\partial u_{J_{1}}^{J_{2}}}
$$

avec

$$
\tau_{J_{2}}^{J_{2}}=\sum_{k=1}^{r} a_{J_{1}, k}^{J_{2}} \omega_{k}^{\prime}
$$

Si on pose:

$$
\tau=\left[\tau_{J_{1}}^{J_{2}}\right]
$$

on a donc: $\tau=a \omega^{\prime}$ et tout revient, pour déterminer explicitement $\tilde{T}^{\prime} \psi_{0}^{1}$, à calculer la matrice $a\left(x^{1}\right)$ pour tout $x^{1} \in O_{i}$. Pour $x^{1}$ donné, $a\left(x^{1}\right)$ est entièrement déterminée par la restriction de l'égalité $\tau=a \omega^{\prime}$ à ${\stackrel{P}{T_{1}}}_{x_{1}}^{\prime}\left(\Phi_{N_{0}} \not \# O_{i}\right)$. Soit $x$ l'origine de $x^{1}$, si l'on identifie ${\stackrel{\circ}{T^{\prime}}}^{\prime}\left(\Phi_{N_{0}} \not O_{i}\right)$ à l'espace $T_{j_{x} 1}\left(\Phi_{x}\right)$ tangent au groupe d'isotropie $\Phi_{x}$ au point $j_{x} 1$, la restriction $\tau_{x^{1}}$ de $\tau$ à ${\stackrel{T}{x^{2}}}^{\prime}\left(\Phi_{N_{0}} \not O_{i}\right)$ s'identifie, par definition même de $\overleftarrow{T}^{\prime} \psi_{0}^{1}$, à la différentielle au point $j_{x} 1$ de l'application $g \rightarrow \varphi_{i, x}\left(g x^{1}\right)$ définie sur $\Phi_{x}$. Cette dermière application étant égale à l'application $g \rightarrow t^{\prime}\left(g^{-1}\right)$ (cf. Lemme 1.7), on se ramène donc à calculer la différentielle $\mathrm{d} t^{\prime}$ de $t^{\prime}$ au point $j_{s} 1 \mathrm{ou}$, ce qui revient même, la restriction à $T_{j_{x} 1} \Phi_{x}$ de la différentielle $d t$ de l'application $t$ définie (cf. $\left.c\right)$ ) sur $\cup \subset \tilde{M}$ par:

$$
\tilde{\eta}^{\prime \prime}=t \tilde{\eta}^{\prime}
$$

En différentiant (3), on a:

$$
d \tilde{\eta}^{\prime \prime}=d t \wedge \tilde{\eta}^{\prime}+t d \tilde{\eta}^{\prime}
$$

En raison de $\left(1^{\prime}\right)$ et $\left(2^{\prime}\right)$, on a donc:

$$
A \tilde{\eta}^{\prime} \wedge \tilde{\eta}^{\prime}+B \tilde{\eta}^{\prime} \wedge \tilde{\omega}=d t \wedge \tilde{\eta}^{\prime}+t\left[\bar{A} \tilde{\eta}^{\prime} \wedge \tilde{\eta}^{\prime}+\bar{B} \tilde{\eta}^{\prime} \wedge \tilde{\omega}\right]
$$

qui s'écrit encore:

$$
A \tilde{\eta}^{\prime} \wedge \tilde{\eta}^{\prime}+B \tilde{\eta}^{\prime} \wedge \tilde{\omega}=d t \wedge \tilde{\eta}^{\prime}+T \bar{A} \tilde{\eta}^{\prime} \wedge \tilde{\eta}^{\prime}+T \bar{B} \tilde{\eta}^{\prime} \wedge \tilde{\omega}
$$

d'où l'on dédnit:

$$
\tilde{\eta}^{\prime} \wedge\left[d t+(A-T \bar{A}) \tilde{\eta}^{\prime}+(B-T \bar{B} \tilde{\omega}]=0\right.
$$

Comme les composantes $\tilde{\eta}_{i_{1}}, \ldots, \tilde{\eta}_{i_{p}}$ de $\tilde{\eta}^{\prime}$ forment un système libre sur $\tilde{M}$, il existe done une matrice $O$ telle que:

$$
d t+(A-T \bar{A}) \tilde{\eta}^{\prime}+(B-T \tilde{B}) \tilde{\omega}=C \tilde{\eta}^{\prime} .
$$


La restriction de cette relation à $T_{j_{x 1}} \Phi_{x}$ s'écrit:

$$
-\tau_{x^{1}}+\left(B\left(x^{1}\right)-T\left(x^{1}\right) \bar{B}\left(x^{1}\right)\right) \omega_{x^{1}}^{\prime}=0,
$$

car

$$
\begin{aligned}
& d t / T_{j_{x} 1} \Phi_{x}=d t^{\prime}=-\tau_{x^{2}} \\
& \tilde{\eta} / T_{j_{x} 1} \Phi_{x}=0 \\
& \tilde{\omega} / T_{j_{x} 1} \Phi_{x}=\omega_{x^{1}}^{\prime}
\end{aligned}
$$

On a done:

$$
a\left(x^{1}\right) \omega_{x^{1}}^{\prime}=\tau_{x^{1}}=\left[B\left(x^{1}\right)-T\left(x^{1}\right) \bar{B}\left(x^{1}\right)\right] \omega_{x^{1}}^{\prime},
$$

d'où

$$
a\left(x^{1}\right)=B\left(x^{1}\right)-T\left(x^{1}\right) \bar{B}\left(x^{1}\right)
$$

Il suffit ensuite d'écrire le système $T l \circ \hat{T}^{\prime} \psi_{0}^{1}=0$ dans les coordonnées $u_{J_{1}}^{J_{z}}$ pour obtenir le résultat.

e) Repèrage absolu à l'ordre 0. - En pratique, e'est la donnée d'un repèrage absolu au-dessus de $N_{0}$ (cf. définition 1.9) qui permet d'obtenir les sections $e$ et $\mu$ ainsi que la forme de pseudo-connexion $\omega$ utilisées en $d$ ).

Soit $T^{\prime}\left(\Phi \# N_{0}\right)$ le fibré des vecteurs tangents à $\Phi \not \# N_{0}$ et verticaux pour la projection de $\Phi \not N_{0}$ sur $N_{0}$. Ce fibré s'identifie à l'ensemble des vecteurs $\alpha$-verticaux de $\alpha^{-1}\left(N_{0}\right)$ lorsqu'on identifie $\Phi \not N_{0}$ à $\alpha^{-1}\left(N_{0}\right) \subset \Phi$. Soit $\stackrel{\circ}{T}^{\prime}\left(\Phi \not N_{0}\right)$ le fibré induit par $T^{\prime}\left(\Phi \not N_{0}\right)$ sur $\Delta_{0}$. On a en particulier $\stackrel{\complement}{T}^{\prime}\left(\Phi_{N_{0}} \# N_{0}\right) \subset \check{T}^{\prime}\left(\Phi \not N_{0}\right)$. On rappelle (cf. $b)$ ) que pour $x \in N_{0},{\stackrel{\circ}{\left.T_{(j x} 1, x\right)}}^{\prime}\left(\Phi_{N_{0}} \not \# N_{0}\right)$ est de dimension $r$.

DÉfinition 1.9. - Un repère absolu en $x \in N_{0}$ est une base

$$
\varepsilon(x)=\left(\varepsilon_{1}(x), \ldots, \varepsilon_{s}(x)\right) \quad \text { de } \quad \stackrel{T}{T}_{\left(j_{x} 1, x\right)}^{\prime}\left(\Phi \# N_{0}\right)
$$

dont les $r$ premiers vecteurs appartiennent $\grave{a ̀ ~}_{\overbrace{(j x 1, x)}^{\prime}}^{\prime}\left(\Phi_{N_{0}} \not \# N_{0}\right)$ (et en constituent done une base).

Un repèrage absolu au-dessus de $N_{0}$ est une famille $\varepsilon_{1}, \ldots, \varepsilon_{s}$ de sections différentiables de $N_{0}$ dans $T^{\prime}\left(\Phi \# N_{0}\right)$ telle que, pour tout $x \in N_{0}, \varepsilon(x)=\left(\varepsilon_{1}(x), \ldots, \varepsilon_{s}(x)\right)$ soit un repère absolu en $x$.

Exemple 1.10. - Soit $G$ le groupe des déplacements de $\boldsymbol{R}^{n}$. L'action de $G$ sur $\boldsymbol{R}^{n}$ étant transitive, on peut prendre $N_{0}=0$. Alors, $\mathscr{T}^{\prime}\left(\Phi \# N_{0}\right)$ est l'algèbre de Lie de $G$ et $\hat{T}^{\prime}\left(\Phi_{N_{0}} \# N_{0}\right)$ l'algèbre de Lie du groupe d'isotropie en $O$ (i.e. $S O(n)$ ).

La donnée d'une base orthonormée de $\boldsymbol{R}^{n}$ (i.e. le repère absolu de $\mathrm{E}$. Cartan) définit un repèrage absolu car, à cette base correspondent des coordonnées $x_{i}$ dans $\boldsymbol{R}^{n}$ et $x_{i}^{J}$ dans $G L(n, \boldsymbol{R})$ et donc des coordonnées $x_{i}, x_{i}^{J}$ dans le groupe affine $\mathcal{A}(n) . S_{\mathrm{j}}$ 
l'on considère dans $T_{\varepsilon} G$ les vecteurs:

$$
\varepsilon_{i}=\frac{\partial}{\partial x_{i}} \text { et } \varepsilon_{i}^{J}=\frac{\partial}{\partial x_{i}^{J}}-\frac{\partial}{\partial x_{J}^{i}},
$$

ils forment une base et définissent un repèrage absolu au-dessus de 0 .

Rappelons (cf. II. A), §1) que $N_{0}$ est l'image d'une section de la $\Phi$-submersion $f_{0}$ de source $U_{0}$, de but $\boldsymbol{R}^{l}$.

Proposition 1.11. - A un repèrage absolu au-dessus de $N_{0}$ sont canoniquement associées des sections différentiables $e$ et $\mu$ de $N_{0}$ dans $H(V)$ et $H\left(I^{\prime}\left(\Phi_{N_{0}} \not N_{0}\right)\right)$ respeetivement et une forme de pseudo-connexion sur $\Phi \# N_{0}$.

Soit en effet $\varepsilon_{1}, \ldots, \varepsilon_{s}$ le repèrage absolu donné. Par définition même, les sections $\varepsilon_{1}, \ldots, \varepsilon_{r}$ définissent une section différentiable $\mu$ de $N_{0}$ dans $H\left(\check{T}^{\prime}\left(\Phi_{N_{0}} \# N_{0}\right)\right)$. D'autre part, les vecteurs $T_{\left(j_{x} 1, x\right)} \psi^{0}\left(\varepsilon_{r+1}\right), \ldots, T_{\left(j_{x} 1, x\right)} \psi^{0}\left(\varepsilon_{s}\right)$ forment une base de l'espace tangent en $x \in N_{0}$ a l'orbite $O_{x}$ de $x$. Si done on désigne par $x_{1}, \ldots, x_{l}$ les coordonnées définies sur $N_{0}$ par la restriction de $f_{0}$ à $N_{0}$ les sections de $T V$ au-dessus de $N_{0}$ définies par:

$$
e_{1}=\frac{\partial}{\partial x_{1}}, \ldots, e_{l}=\frac{\partial}{\partial x_{l}}, e_{l+1}=T \psi^{0}\left(e_{r+1}\right), \ldots, e_{n}=T \psi^{0}\left(\varepsilon_{s}\right)
$$

définissent une section différentiable $e$ de $N_{0}$ dans $H(V)$.




Il reste à déterminer la forme de pseudo-connexion. Soit $\stackrel{T}{T}\left(\Phi \# N_{0}\right)$ le fibré induit sur $\Delta_{0}$ par le fibré tangent $T\left(\Phi \# N_{0}\right)$ et soit $E$ le sous-fibré de $T\left(\Phi \# N_{0}\right)$ engendré par $T \Delta_{0}$ et les images des sections $\varepsilon_{r+1}, \ldots, \varepsilon_{s}$. On a:

$$
\stackrel{\circ}{T}\left(\Phi N_{0}\right)=E \oplus \stackrel{\circ}{ }^{\prime}\left(\Phi_{N_{0}} \not N_{0}\right)
$$

On désigne par $\Pi$ la projection de $\stackrel{T}{T}\left(\Phi \not N_{0}\right)$ sur $\stackrel{T}{ }^{\prime}\left(\Phi_{N_{0}} \# N_{0}\right)$ définie par cette décomposition en somme directe. Soit alors $\omega: T \Phi \rightarrow \stackrel{\circ}{\Phi}$ la forme canonique sur $\Phi$ définie au (I. A), §2). En identifiant $\Phi \not N_{0}$ à une sous-variété de $\Phi$ par le plongement $\left(j_{x} \varphi, x\right) \rightarrow j_{x} \varphi$, la restriction de $\omega$ à $T\left(\Phi \# N_{0}\right)$ définit un morphisme ancore noté $\omega$, de $T\left(\Phi \# N_{0}\right)$ dans $\stackrel{T}{T}\left(\Phi \# N_{0}\right)$. On vérifie alors que $\Pi_{\circ} \omega: T\left(\Phi \# N_{0}\right) \rightarrow$ $\rightarrow i^{\prime}\left(\Phi_{N_{0}} \# N_{0}\right)$ est une forme de pseudo-connexion sur $\Phi \not N_{0}$.

EXEMPLE 1.12. - En reprenant l'exemple du groupe $G$ des déplacements de $R^{n}$, la section $\mu$, qui se réduit ici à $\mu(0)$, est la base $\partial / \partial x_{i}^{J}-\partial / \partial x_{i}^{J}$ de l'algèbre de Lie $O(n)$. La section e, définie par e(0) est la base de $\boldsymbol{R}^{n}$ définie par le repère absolu. Enfin, la forme de pseudo-connexion est obtenue en composant la forme invariante à gauche $T G \rightarrow T_{\varepsilon} G$ avec la projection de $T_{\varepsilon} G$ sur o(n) définie par la décomposition $T_{\varepsilon} G=$ $=o(n) \oplus \boldsymbol{R}^{n}$ associée aux coordonnées.

Remarque 1.13. - Lorsque, partant d'un repèrage absolu, on utilise la règle de E. Cartan, la détermination des matrices $A, B, \bar{A}$ et $\bar{B}$ définies au d) est simple une fois connues les équations de structure du groupoïde $\Phi$ (i.e. de la forme canonique $\omega$ ).

\section{§2. Passage de l'ordre 0 à l'ordre 1.}

Dans la paragraphe 1 , le fait que l'ouvert saturé $U_{0}$ soit un ouvert de la base du groupoïde $\Phi$ est essentiel. Pour pouvoir reconduire la méthode à l'ordre 1 , on doit done se ramener à une situation du même type; ceci est l'objet du a) ei-dessous. On montre ensuite au $b$ ) comment à partir d'un repèrage absolu à l'ordre 0 il est possible, sans données supplèmentaires, d'obtenir un repèrage absolu à l'ordre 1.

a) Le groupoïde $\Phi^{c}$. - Soit $\Gamma^{c}$ le pseudogroupe engendré dans $C P^{1}$ par les difféomorphismes locaux $\varphi^{1, c}$ de $C P^{1}$ obtenus en prolongeant les transformations $\varphi \in T$ (cf. I, B), $\S 1, b)$ ). On vérifie aisément que, de même que $\Gamma, \Gamma^{c}$ est un pseudogroupe de Lie de type fini et que son groupoïde de définition, qu'on note $\Phi^{\circ}$, s'identifie au groupoïde $\Phi \neq C P^{1}$ des elements composables pour l'action de $\Phi$ dans $C P^{1}$. La base du groupoïde $\Phi^{c}$ est $C P^{1}$; on note $\alpha^{c}$ et $\beta^{c}$ les applications source et but de $\Phi^{e}$ sur $C P^{1}$.

Soit $\widetilde{C P^{2}}=C P^{1}\left(C P^{1}\right)$ la variété des éléments de contact de dimension $p$ de $C P^{1}$. Le groupoïde $\Phi^{\circ}$ opère naturellement dans $\widetilde{O P^{2}}$ et l'on pent utiliser, pour la recherche des invariants différentiels pour cette action de $\Phi^{c}$ dans $\tilde{C} P^{a}$ la méthode du paragraphe précédent. 
La variété $C P^{2}$ des éléments de contact de dimension $p$ et d'ordre 2 de $V$ se plonge canoniquement dans $\tilde{C} P^{2}$. Plus précisément. avec les notations du $\left.(\mathrm{I}, \mathrm{B}), \S 1\right)$, soit $\bar{j}_{0}^{2} \mu \in C P^{2}$, et soit $\bar{\mu}$ l'application de $\boldsymbol{R}^{p}$ dens $C P^{1}$ définie par: $\vec{\mu}(x)=\bar{j}_{0}\left(\mu \circ \tau_{x}\right)$. $\bar{\mu}$ est une immersion et $\bar{j}_{0}^{1} \bar{\mu}$ est un élément de $\tilde{C} P^{2}$ ne dépendant que de $\bar{j}_{0}^{2} \mu$ : c'est l'image de $\bar{j}_{0}^{2} \mu$ par le plongement canonique.

On vérifie ensuite aisément que $C P^{2}$ est stable pour l'action de $\Phi^{a}$ dans $\widetilde{C P}^{2}$ et que pour l'action ainsi induite de $\Phi^{c}$ dans $C P^{2}$ les orbites sont les mêmes que pour laction de $\Phi$ dans $C P^{2}$. Ceci montre que les invariants differentiels pour l'action de $\Phi$ dans $C P^{2}$ sont les restrictions à $C P^{2}$ des invariants différentiels pour l'action de $\Phi^{\circ}$ dans $\tilde{C} P^{\text {s. }}$. La règle de $\mathrm{E}$. Cartan peut done encore être utilisée pour la recherche des invariants différentiels d'ordre 2. Plus généralement, pour les mêmes raisons, cette règle pourra être utilisée pour la détermination des invariants d'ordre $n$, une fois connus ceux d'ordre $n-1$.

b) Repèrage absolu à l'ordre 1. - Les données sont les suivantes:

$f_{0}$

$N_{0}: \quad$ image d'une section différentiable de $f_{0}$.

$f_{1}: \quad \Phi^{c}$-submersion définie sur un ouvert de $O P^{1}$.

$N_{1}$ : $\quad$ image d'une section différentiable de $f_{1}$. On suppose que $N_{1}$ se projette sur $N_{0}$ par la projection de $C P^{1}$ sur $V$.

On adopte, pour le groupoïde $\Phi^{\circ}$, la même méthode de notation que pour $\Phi$, ainsi:

$\Phi^{c} \not N_{1}: \quad$ est le produit fibré de $\Phi^{\circ}$ et $N_{1}$ pour les applications $\alpha^{c}: \Phi^{\circ} \rightarrow N_{1}$ et $1: N_{1} \rightarrow N_{1}$.

$\Phi_{N_{1}}^{c}$ : $\quad$ est le groupoïde d'isotropie de $\Phi^{c}$ au-dessus de $N_{1}$; c'est done la réunion pour $x_{1} \in N_{1}$ des groupes d'isotropies $\Phi_{x^{1}}^{c}$.

$\Phi_{N_{1}}^{c} \# N_{1}$ : est le produit fibré de $\Phi_{N_{1}}^{c}$ et $N_{1}$ pour les projections canonique de $\Phi_{N_{1}}^{c}$ et $N_{1}$ sur $N_{1}$. On $a: \Phi_{N_{1}}^{c} \not N_{1} \subset \Phi^{\circ} \not N_{1}$.

$\Delta_{1}: \quad \quad$ est l'ensemble des éléments de $\Phi^{c} \not N_{1}$ de la forme $\left(j_{x^{1}} 1, x^{1}\right)$.

$\check{T}^{\prime}\left(\Phi^{\circ} \# N_{1}\right)$ : est le fibré induit sur $\Delta_{1}$ par le fibré des vecteurs tangents à $\Phi^{c} \not N_{1}$ et verticaux pour la projection de $\Phi^{\circ} \not N_{1}$ sur $N_{1}$.

$\stackrel{\circ}{T}^{\prime}\left(\Phi_{N_{0}}^{c} \# N_{1}\right)$ : est le sous-fibré de $\grave{T}^{\prime}\left(\Phi^{o} \# N_{1}\right)$ formé des vecteurs tangents à $\Phi_{N_{0}}^{c} \# N_{1}$ et verticaux pour la projection sur $N_{1}$.

Plaçons-nous d'abord en un point $x^{1} \in N_{1}$ d'origine $x \in N_{0}$. On a une identification canonique de $\left(\alpha^{c}\right)^{-1}\left(x^{1}\right) \subset \Phi^{c}$ avec $\alpha^{-1}(x) \subset \Phi$. Par cette identification, le groupe d'isotropie $\Phi_{x^{1}}^{c}$ de $\Phi^{c}$ en $x^{1}$ s'identifie à un sousgroupe de $\Phi_{x}$; groupe d'isotropie de $\Phi$ en $x$. De plus, avec les notations du (II, A), §1), le sous-groupe de $\Phi_{x}$ ainsi obtenu est l'image réciproque de $x^{1}$ par l'application $\psi_{0}^{1}$ (ou plutot sa restriction à $\Phi_{x} \times\left\{x^{1}\right\}$ 
qu'on identifie à $\left.\Phi_{x}\right)$. L'algèbre de Lie de $\Phi_{x^{1}}^{c}$ considéré comme sous-groupe de $\Phi_{x}$ est donc le noyau de la restriction de $\hat{T}^{\prime} \psi_{0}^{1}$ à la fibre de $\mathscr{T}^{\prime}\left(\Phi_{N_{0}} \# N_{1}\right)$ andessus de $\left(j_{x} 1, x^{1}\right)$ c'est-à-dire à l'algèbre de Lie de $\Phi_{x}$.

Ceci étant, en $x \in N_{0}$, comme en $x^{1} \in N_{1}$, un repère absolu est une base d'un certain type de l'espace tangent à $\alpha^{-1}(x)$ au point $j_{x} 1$. La différence est essentiellement la suivante: dans le premier cas, les $r$ premiers vecteurs doivent former une base de l'algèbre de Lie de $\Phi_{x}$, dans le second cas, les $r_{1}$ premiers vecteurs doivent former une base de l'algèbre de Lie de $\Phi_{x^{1}}^{c}$ (avec $r_{1}=\operatorname{dim} \Phi_{x^{1}}^{c}$ ).

La méthode pour obtenir un repère absolu d'ordre 1 à partir d'un repère absolu d'ordre 0 est maintenant claire. On doit, par combinaisons linéaires de certains vecteurs du repère d'ordre 0 , obtenir une base de l'algèbre de Lie de $\Phi_{x^{1}}^{c}$ et compléter cette base par des vecteurs du repère d'ordre 0 pour former une base de $T_{j_{z} 1}\left(\alpha^{-1}(x)\right)$.

Le passage au global se fait sans difficultés. Soit $\Phi \# N_{1}$ (resp. $\Phi_{N_{0}} \# N_{1}$ ) le produit fibré de $\Phi \not N_{0}$ (resp. $\Phi_{N_{0}} \# N_{0}$ ) et $N_{1}$ sur $N_{0}$. Avec les notations du (II, A), §1), on a: $\Phi_{N_{0}} \# N_{1} \subset \Phi_{N_{0}} \# N_{0}^{1}$. On convient de noter encore $\stackrel{\circ}{T}^{\prime} \psi_{0}^{1}$ la restriction de $\stackrel{\circ}{T}^{\prime} \psi_{0}^{1}$ à $\stackrel{\circ}{T}^{\prime}\left(\Phi_{N_{0}} \# N_{1}\right)$, fibré inđuit sur $\Phi_{N_{0}} \# N_{1}$ par $T^{\prime}\left(\Phi_{N_{0}} \# N_{0}^{1}\right)$.

LeMme 2.1. - L'application $\left(j_{x} \varphi, x, x^{1}\right) \rightarrow\left(j_{x} \varphi, x^{1}, x^{1}\right)$ de $\Phi \times N_{0} \times N_{1}$ dans $\Phi \times$ $\times N_{1} \times N_{1}$ induit un difféomorphisme de $\Phi \not N_{1}$ sur $\Phi^{c} \# N_{1}$ compatible avec les projections de $\Phi \# N_{1}$ et $\Phi^{c} \not N_{1}$ sur $N_{1}$.

Démonstration évidente. - Ce lemme permet d'identifier $\stackrel{\circ}{T}^{\prime}\left(\Phi^{\circ} \# N_{1}\right)$ avec l'espace $i^{\prime}\left(\Phi \# N_{1}\right)$ des vecteurs tangents à $\Phi \# N_{1}$ aux points de la forme $\left(j_{x} 1, x^{1}\right)$ et verticaux pour la projection de $\Phi \# N_{1}$ sur $N_{1}$.

LEMME 2.2. - Par l'identification de $\Phi^{c} \# N_{1}$ avec $\Phi \not N_{1}, \Phi_{N_{1}}^{c} \# N_{1}$ est contenu dans $\Phi_{N_{0}} \not \# N_{1}$ et $\stackrel{\circ}{T}^{\prime}\left(\Phi_{N_{1}}^{c} \not N_{1}\right)=\operatorname{Ker}\left(\stackrel{T}{T}^{\prime} \psi_{0}^{1}\right)$.

Pour démontrer ce lemme, on procède fibre par fibre. Et pour chaque fibre, il suffit d'utiliser la même méthode que celle utilisée plus haut au point $x^{1} \in N_{\text {. }}$.

Corollatre 2.3. - Un repèrage absolu au-dessus de $N_{1}$ est une famille de $s$ sections différentiables indépendantes de $N_{1}$ dans $\hat{T}^{\prime}\left(\Phi \not N_{1}\right)$ dont les $r_{1}$ pemières sont à valeurs dans $\operatorname{Ker} \stackrel{\circ}{T}^{\prime} \psi_{0}^{1}$.

Evident.

Soit $\varepsilon_{1}, \ldots, \varepsilon_{s}$ un repèrage absolu au-dessus de $N_{0}$, soient $e$ et $\mu$ les sections de $N_{0}$ dans $H(V)$ et $H\left(\hat{T}^{\prime}\left(\Phi \not N_{0}\right)\right)$ respectivement et $\omega$ la forme de pseudo-connexion associées (cf. proposition 1.11).

En utilisant les résultats et notations $d \mathrm{~d} \xi 1, b)$, soit $\omega_{1}^{\prime}, \ldots, \omega_{r}^{\prime}$ la base des morphismes $\stackrel{\circ}{T}^{\prime}\left(\Phi_{N_{0}} \not \# N_{0}^{1}\right) \rightarrow \boldsymbol{R}$ associée à $\omega$. La restriction de $\stackrel{O}{T}^{\prime} \psi_{0}^{1}$ à $\stackrel{\circ}{T}^{\prime}\left(\Phi_{N_{0}} \# N_{1}\right)$ s'exprime par:

$$
\tau_{J_{3}}^{J_{2}}=\sum_{k=1}^{r} a_{J_{1} k}^{J_{3}} \omega_{k}^{\prime} \quad J_{1}=1, \ldots, p \quad J_{2}=1, \ldots,(n-p)
$$

et comme $\operatorname{Ker}\left(\grave{T}^{\prime} \psi_{0}^{1}\right)$ a des fibres de dimension $r_{1}$, par hypothèse, il existe parmi les formes $\tau_{J_{1}}^{J_{2}}, r-r_{1}$ formes linéairement indépendantes, qu'on notera $\tau^{J_{r_{1}+1}}, \ldots, \tau^{J_{r}}$. 
Complétons ce système par des formes $\omega_{k_{1}}^{\prime}, \ldots, \omega_{k_{r_{1}}}^{\prime}$ de sorte que:

$$
\omega_{k_{1}}^{\prime}, \ldots, \omega_{k_{r_{i}}}^{\prime}, \quad \tau^{J_{r_{1}+1}}, \ldots, \tau^{J_{r}}
$$

soit une base de l'espace des formes $\stackrel{\circ}{T}^{\prime}\left(\Phi_{N_{0}} \# N_{1}\right) \rightarrow \boldsymbol{R}$. Soit $\varepsilon_{1}^{c}, \varepsilon_{2}^{c}, \ldots, \varepsilon_{r_{1}}^{c}, \varepsilon_{r_{1}+1}^{c}, \ldots$, $\varepsilon_{r}^{c}$ la base duale. Les $\varepsilon_{i}^{c}$ sont des sections differentiables de $N_{1}$ dans $\hat{T}^{\prime}\left(\Phi_{N_{0}} \not N_{1}\right)$ vérifiant les conditions suivantes pour tout $x^{1} \in N_{1}$ :

1) $\varepsilon_{1}^{c}\left(x^{1}\right), \ldots, \varepsilon_{r}^{c}\left(x^{1}\right)$ est une base de ${\stackrel{\circ}{\left(j_{x} 1, x^{1}\right)}}_{\left(N_{0}\right.} \# N_{1})$;

2) $\varepsilon_{1}^{c}\left(x^{1}\right), \ldots, \varepsilon_{r_{1}}^{c}\left(x^{1}\right)$ est à valeurs dans $\operatorname{Ker}\left(\stackrel{T}{T}^{\prime} \psi_{0}^{1}\right)$.

Pour obtenir un repèrage absolu à l'ordre 1 , on doit compléter le système $\varepsilon_{1}^{c}, \ldots, \varepsilon_{r}^{c}$. On utilise pour cela la remarque suivante: la projection de $\Phi \# N_{1}$ sur $\Phi \# N_{0}$ définie par $\left(j_{x} \varphi, x^{1}\right) \rightarrow\left(j_{x} \varphi, x\right)$ induit pour chaque $x^{1} \in N_{1}$ un isomorphisme de $\stackrel{\sigma}{T}_{\left(j_{x} 1_{1} x_{1}\right)}^{\prime}\left(\Phi \not N_{1}\right)$ sur $\stackrel{\circ}{T}_{\left(j_{x} 1, x\right)}^{\prime}\left(\Phi \# N_{0}\right)$; on définit done pour chaque $i$ une section différentiable $\tilde{\varepsilon}_{i}$ de $N_{1}$ dans $T^{\prime}\left(\Phi \# N_{1}\right)$ telle que le diagramme suivant est commutatif:

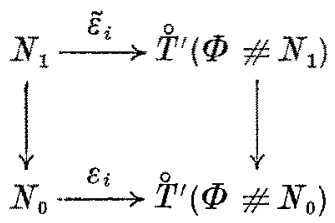

Ceci étant, par définition même d'un repèrage au-dessus de $N_{0}$, pour chaque $x^{1} \in N_{1}$, l'espace engendré par $\tilde{\varepsilon}_{1}\left(x^{1}\right), \ldots, \tilde{\varepsilon}_{r}\left(x^{1}\right)$ est ${\stackrel{T}{\left(j_{z} 1, x^{1}\right)}}^{\prime}\left(\Phi_{N_{0}} \not \# N_{1}\right)$, e'est done l'espace engendré par $\varepsilon_{1}^{c}\left(x^{1}\right), \ldots, \varepsilon_{y}^{c}\left(x^{1}\right)$. En posant:

$$
\varepsilon_{k}^{c}=\tilde{\varepsilon}_{k} \quad \text { pour } k=r+1, \ldots, s
$$

on voit que $\varepsilon_{1}^{c}, \ldots, \varepsilon_{s}^{c}$ est un repèrage absolu au-dessus de $N_{1}$. Le passage annoncé d'un repèrage absolu à l'ordre 0 à un repèrage absolu à l'ordre 1 est ainsi réalisé. Le lecteur vérifiere en outre que ce repèrage est bien adapté à la poursuite des calculs.

\section{BIBLIOGRAPHIE}

[1] BoURBaKI. Variétés différentielles et analytiques.

[2] E. CARTAN, La théorie des groupes finis et continus et la géométrie différentielle traitées par la méthode du repère mobile, Ganthier-Villars, 1951.

[3] C. Errmasmann, Colloque de géométrie différentielle, Strasbourg, 1953.

[4] C. Ehresmant, Sur les pseudogroupes de Lie de type fini, Compt. Rend, Acad. Sei., 1958.

[5] J. Favard, Cours de géométrie différentielle locale, Gauthier-Villars, 1957.

[6] V. Guthlemin - S. Sternberg, Deformation theory of pseudogroup structures, Memoirs of the Am. Math. Soc., vol. 64.

[7] P. Lrbarmanv, Thèse, Strasbourg, 1953; Annali di Matematica, 36 (1954).

[8] P. LIBERMANn, Sur les groupondes différentiables et le presque parallélisme, Istituto Nazionale di Alta Matem. Symposia Mat., Roma, 1971. 